\title{
Feasibility of Monte Carlo modelling for the neutron-neutron logging tool response in specific geological models
}

\author{
Urszula Wiącek, Urszula Woźnicka \\ Institute of Nuclear Physics Polish Academy of Sciences, Krakow, Poland \\ (C) 2016 Authors. This is an open access publication, which can be used, distributed and reproduced in any medium according \\ to the Creative Commons CC-BY 4.0 License requiring that the original work has been properly cited.
}

Received: 28 April 2016; accepted: 9 June 2016

\begin{abstract}
Neutron well logging is one of the basic methods for the determination of the characteristic parameters of rock samples. The neutron source and neutron detectors are elements of Neutron-Neutron Thermal-Epithermal logging tool (NNTE) of significant importance. A neutron source creates the neutron field in the nearest environment. Detectors placed at specified distances from the source register neutrons from this space. A signal of a Neutron-Neutron Thermal-Epithermal tool in specific geological conditions was numerically calculated by means of the Monte Carlo (MC) codes. The main aim of this paper is to show the potential for using the Monte Carlo N-Particle Transport Code (MCNP) software in nuclear well logging prospection methods. The results of this MC modelling are presented in this paper.
\end{abstract}

Keywords: Monte Carlo calculations; neutron well logging; NNTE density tool; neutron parameters

\section{INTRODUCTION}

The neutron logging probe contains the neutron source and a set of detectors which register neutrons after their propagation in the vicinity of the borehole. The neutron source emits fast neutrons and a neutron field is created in a surrounding matter, i.e. in a whole space which encompasses the borehole and surrounding geological layers. At each collision the fast neutron transfers a significant part of its kinetic energy to the scattering nucleus; greater if the nucleus is lighter. It means that most of these interactions are related to the hydrogen content present in the medium. Neutrons are slowed down, thermalized, and finally are absorbed in the matter. In these considerations the most important parameters of neutron interactions with matter are scattering and absorption on nuclei of the medium in which neutrons are propagated. Neutron scattering and absorption cross sections depend on neutron energy and widely vary from isotope to isotope. Detectors in a neutron probe register thermal and/or epithermal neutrons, i.e. those neutrons which have lost their energy during propagation in the vicinity of the borehole. The set of thermal and epithermal neutron detectors is the base of the "neutron porosity tool". Neutrons which reach the system of detectors bring complex information with regards to rock characteristics. The measured signals permits the calculation of one of the most important petrophysical parameters of the formation, i.e. porosity, and in consequence, resources of hydrocarbon deposits.

\section{THE NNTE BOREHOLE NEUTRON TOOL}

One technical solution for the need for a borehole neutron tool is the NNTE (Neutron-Neutron Thermal-Epithermal) probe which is designed 
to measure porosity and the thermal neutron absorption cross section $\left(\Sigma_{a}\right)$ of rock matrix in oil and gas prospecting wells (Zorski \& Stadtmüller 2001). The probe is equipped with a fast neutron source (Am-Be), two "near" detectors (thermal and epithermal) and one "far" epithermal neutron detector. The proper set of cadmium and neutron moderators is placed around the detectors. They determine that Bepi and Depi detectors measure mainly epithermal neutron flux and the Bter detector is sensitive only to thermal neutrons from the geological layer. Information about porosity is derived from readings of the "near" detectors or from the ratio of the "near" to "far" epithermal detector readings. Information about the rock matrix $\Sigma_{a}$ is derived from the difference between the neutron porosities obtained from the "near" thermal and epithermal detectors.

The general diagram of the geometry of the NNTE tool with the location of neutron source and positions of the detectors are shown in
Figure 1 (dimensions in centimeters). The neutron counters are filled with helium $\left({ }^{3} \mathrm{He}\right)$. Signals registered by the counters are induced by protons generated in the reaction of neutron and helium: ${ }^{3} \mathrm{He}(\mathrm{n}, \mathrm{p})^{3} \mathrm{H}$. The proper design of the detection system ensures that thermal and epithermal neutrons are recorded in the respective counters. The NNTE probe contains an isotopic neutron source made from a mixture of beryllium $\left({ }^{9} \mathrm{Be}\right)$ and americium $\left({ }^{241} \mathrm{Am}\right)$ as an alpha emitter. Am-Be mixture is enclosed in a tight steel container so that the alpha particles do not escape outside. Neutrons are created in reaction between ${ }^{9} \mathrm{Be}$ and alpha particles. Beryllium powder bombarded with alpha particles emits neutron flux. The neutron energy spectrum of the Am-Be neutron source extends up to $11 \mathrm{MeV}$ with the maximum around $3 \mathrm{MeV}$. Average neutron energy is about $4.5 \mathrm{MeV}$. The detailed data for the energy spectrum which are needed as the input data to the MCNP calculations are taken from Klüge (1998) and Drabina et al. (2003).

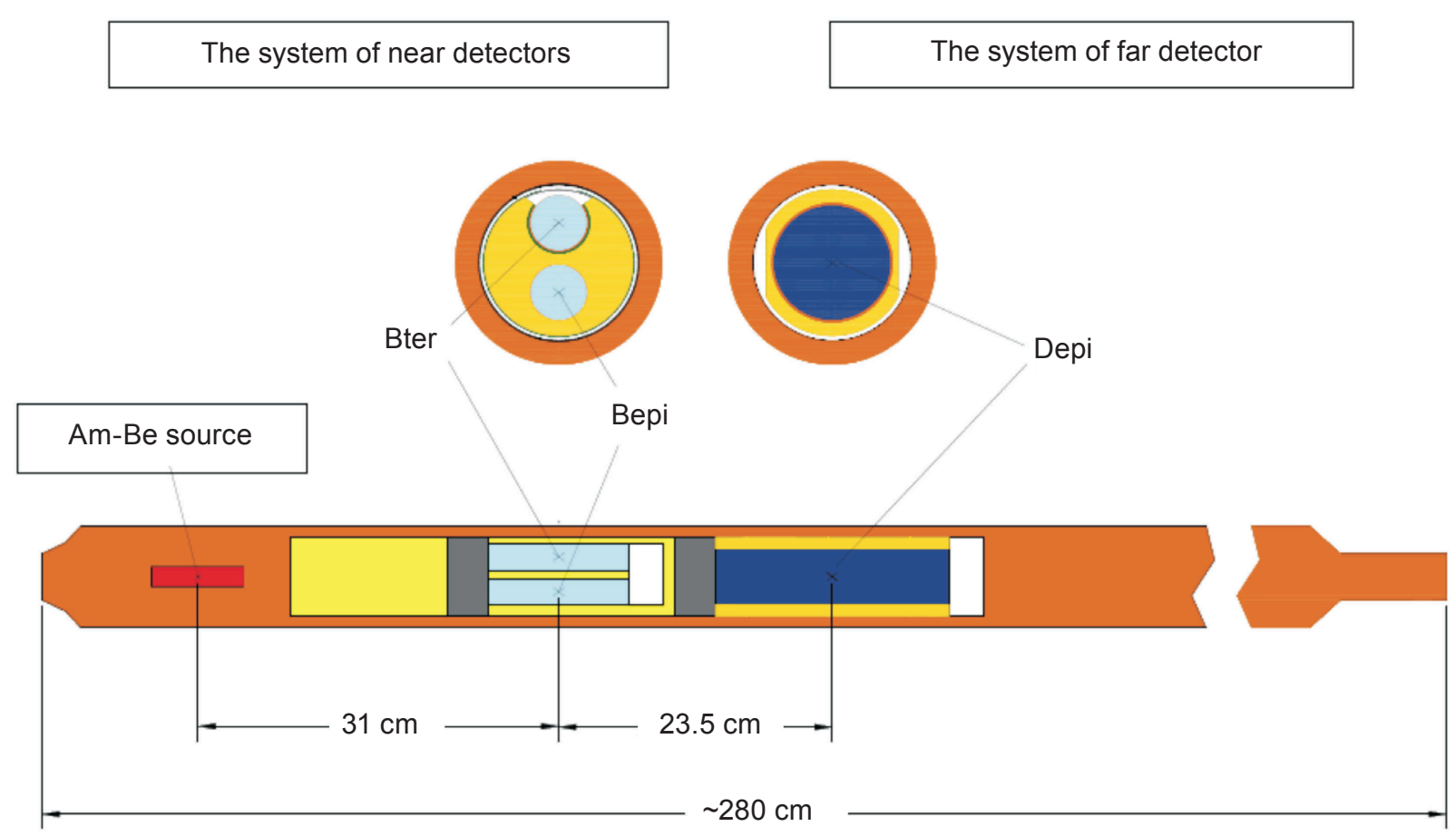

Fig. 1. Sketch geometry of the NNTE tool 


\section{MONTE CARLO CALCULATIONS FOR SIMULATIONS OF NEUTRON PROBE RESPONSE}

Monte Carlo calculations are a convenient way to model and accurately simulate the actual experimental conditions of neutron well logging measurements. We use the Monte Carlo N-Particle Transport code (version MCNP5) (MCNP team 2008) for these applications. The program simulates a random transport of neutrons and other particles in media of a complex composition and geometry based on Monte Carlo method. The whole history of the particular particle is observed from the creation in the user defined source to the absorption in the matter (e.g. in a detector of the probe). This is a powerful tool for providing information of neutron measurements in complex borehole conditions. Parameters such as elemental composition of rock, porosity, geometry of the borehole, scheme of construction of the tool is provided to the program as the input data. All parameters can be modified in an easy way according to needs and it is possible to check the influence of them on the detector response.

The geometry in the MCNP program is built in a three-dimensional configuration of cells bound by first- or second-degree surfaces. The cells are defined by intersections and unions of these surfaces. Each cell is filled with the material of a given isotopic composition of elements. For each material, the mass or atomic density has to be defined by user. The probability of a particle behavior is related to the nuclear cross sections and depends on isotopic composition and the material density of the matter. In the foregoing calculations, the cross sections were taken mainly from the ENDF/B-VI library (Herman \& Trkov 2009).

In MCNP, the tally cards are used to specify the type of information the user wants to gain from the Monte Carlo calculation. The parameters implicated in these cards are used to describe tally "bins", subdivisions of the tally space into discrete and contiguous increments such as cosine, energy, or time. The Surface Tally Type (f4) i.e. particle flux averaged over a cell (track length estimate of cell flux) for neutrons in conjunction with the Tally Multiplier card ( $f m$ card) was used in the performed calculations. The Tally Multiplier card permits one to obtain the total number of absorption reactions in the volume of each detector in the whole energy range. The $\mathrm{MC}$ calculations have been done for $2 \times 10^{9}$ neutrons emitted from the source. The maximum relative error for these calculations was less than $5 \%$. To elaborate and present the results of the MC calculations the MATLAB program (R2012b) has been used (The MathWorks INC. 2012).

\section{MCNP BENCHMARK CALCULATIONS}

To validate the numerical calculations the benchmark procedure has been done. The set of numerical calculations corresponding to the real experiments realized at the calibration facility in Zielona Góra have also been conducted. Based on the results obtained at the IFJ PAN by Andrzej Drabina, the correlation between measurements and calculations has been carried out.

The facility in Zielona Góra is equipped with over a dozen rock models of different lithology: sandstones, limestones, dolomites of variable porosities. Rock pores are $100 \%$ saturated with fresh water. Models are placed in a water pool with a thick concrete floor. Each rock model has a vertical water filled borehole with a diameter $145 \mathrm{~mm}$ or $220 \mathrm{~mm}$. The porosity, density and chemical compositions of the rock matrices are known from laboratory analyses (Zorski et al. 1996). The data are presented in Tables 1 and 2.

Figure 2 shows the geometry of the NNTE tool configuration in the calibration block used for the MCNP simulations and for the real measurements. The probe is placed inside the block in such a way, that the source is in the middle of the block height. The probe is decentralized and adjacent to the wall of the hole. The dimensions used in the calculation are as follows: the thickness of the concrete layer below the block: $1.5 \mathrm{~m}$, the thickness of the water layer above the block: $0.5 \mathrm{~m}$ and thickness of the water layer surrounding the block: $1.2 \mathrm{~m}$.

For each nuclide, the nuclear data for the description of neutron transport have been taken from the relevant specialized data libraries. The ENDFB-VI.6 and ENDFB-VI.2 neutron data libraries (Herman \& Trkov 2009) were used in the first approach together with the data of elemental composition as in Table 1, i.e., the presence of strong absorbents of neutrons was not included (Drabina et al. 2003). 


\begin{tabular}{|c|c|c|c|c|c|c|c|c|c|c|c|c|c|c|c|c|c|c|}
\hline 赵 & $\begin{array}{l}\vec{J} \\
\text { D } \\
\text { i }\end{array}$ & $\begin{array}{l}\infty \\
\stackrel{\infty}{0} \\
i \\
i\end{array}$ & $\mid \begin{array}{l}0 \\
\dot{-} \\
\end{array}$ & $\begin{array}{l}\text { L } \\
0 \\
\\
0 \\
0 \\
0 \\
0\end{array}$ & 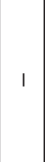 & 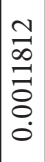 & 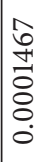 & 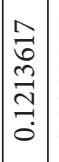 & 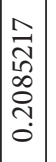 & \begin{tabular}{|c|}
$\infty$ \\
$\infty$ \\
0 \\
$\tilde{\delta}$ \\
0 \\
0 \\
0
\end{tabular} & $\left|\begin{array}{l}\infty \\
\infty \\
0 \\
0 \\
0 \\
0 \\
0 \\
0\end{array}\right|$ & 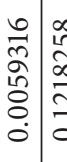 & 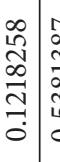 & 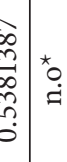 & 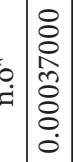 & $\mid$\begin{tabular}{l|}
0 \\
0 \\
0 \\
0 \\
0 \\
0 \\
0 \\
0 \\
0 \\
0
\end{tabular} & \begin{tabular}{|l}
$\star$ \\
0 \\
$\dot{g}$
\end{tabular} & ^ \\
\hline 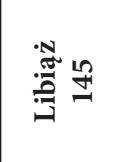 & $\begin{array}{l}\widetilde{Z} \\
\infty \\
i \\
i\end{array}$ & $\begin{array}{l}\text { ్ָర } \\
i \\
i\end{array}$ & $\exists$ & 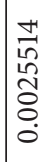 & 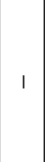 & 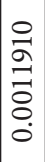 & 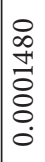 & 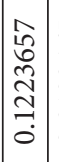 & 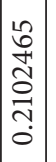 & \begin{tabular}{|c|}
\multirow{2}{*}{} \\
$\infty$ \\
$\widetilde{0}$ \\
0 \\
0 \\
0 \\
0
\end{tabular} & $\mid \begin{array}{l}2 \\
2 \\
\hat{0} \\
0 \\
0 \\
0 \\
0 \\
0\end{array}$ & 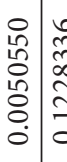 & 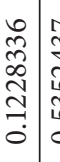 & & $=$ & $\mid$\begin{tabular}{l|}
0 \\
0 \\
0 \\
0 \\
0 \\
0 \\
0 \\
0 \\
0
\end{tabular} & \begin{tabular}{|l}
$\star$ \\
0 \\
$\dot{g}$
\end{tabular} & 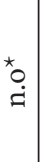 \\
\hline 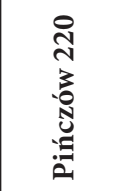 & $\begin{array}{l}\text { 苛 } \\
\text { i }\end{array}$ & $\begin{array}{l}\text { Oे } \\
\stackrel{\vec{i}}{i}\end{array}$ & $\left|\begin{array}{l}\infty \\
\dot{p} \\
\dot{m}\end{array}\right|$ & 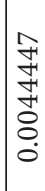 & । & $\begin{array}{l}0 \\
0 \\
0 \\
0 \\
0 \\
\vdots \\
0 \\
0\end{array}$ & $\begin{array}{l}\text { H } \\
0 \\
0 \\
0 \\
0 \\
0 \\
0\end{array}$ & $\left|\begin{array}{c}0 \\
0 \\
\hat{N} \\
\tilde{\delta} \\
0 \\
0 \\
0\end{array}\right|$ & 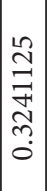 & 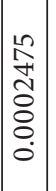 & $\left|\begin{array}{l}n \\
\infty \\
0 \\
0 \\
0 \\
0 \\
0 \\
0\end{array}\right|$ & \begin{tabular}{|l|l}
$\vec{T}$ & $\alpha$ \\
0 & $\alpha$ \\
$\infty$ & $\alpha$ \\
0 & 0 \\
0 & 0 \\
0 & 0 \\
0 & 0
\end{tabular} & $\begin{array}{l}0 \\
\infty \\
\infty \\
\infty \\
0 \\
0 \\
0 \\
0\end{array}$ & 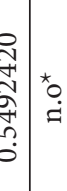 & $=$ & 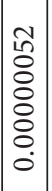 & \begin{tabular}{|c|}
0 \\
0 \\
1 \\
0 \\
0 \\
0 \\
0 \\
0 \\
0 \\
0 \\
0
\end{tabular} & $\begin{array}{l}\text { ôे } \\
\text { ఏे } \\
\vdots \\
\vdots \\
0 \\
0\end{array}$ \\
\hline 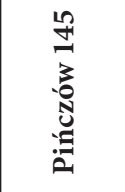 & $\underset{\substack{0 \\
i}}{\stackrel{0}{i}}$ & $\begin{array}{l}\vec{D} \\
0 \\
0 \\
i\end{array}$ & $\left|\begin{array}{l}\infty \\
0 \\
\dot{b} \\
m\end{array}\right|$ & 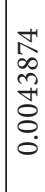 & I & $\begin{array}{l}8 \\
0 \\
0 \\
0 \\
0 \\
0 \\
0\end{array}$ & \begin{tabular}{|l}
0 \\
0 \\
0 \\
0 \\
0 \\
0 \\
0 \\
0
\end{tabular} & 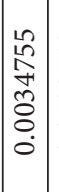 & 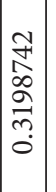 & 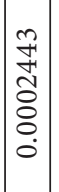 & $\mid \begin{array}{l}1 \\
0 \\
m \\
0 \\
0 \\
0 \\
0\end{array}$ & 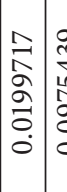 & 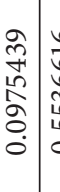 & 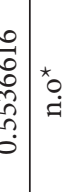 & 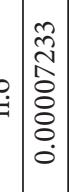 & 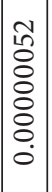 & 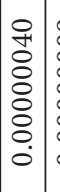 & 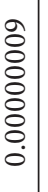 \\
\hline 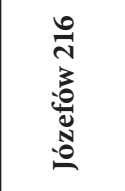 & $\begin{array}{l}0 \\
0 \\
0 \\
i \\
i\end{array}$ & $\begin{array}{l}\vec{H} \\
\stackrel{H}{+} \\
i \\
i\end{array}$ & $\mid \begin{array}{l}\hat{A} \\
\vec{a} \\
\dot{2}\end{array}$ & $\mid \begin{array}{l}\text { } \\
\infty \\
0 \\
0 \\
0 \\
0 \\
0\end{array}$ & I & $\begin{array}{l}\overrightarrow{1} \\
0 \\
0 \\
0 \\
0 \\
0 \\
0\end{array}$ & 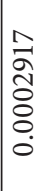 & $\mid \begin{array}{c}\hat{\alpha} \\
\tilde{F} \\
\stackrel{+}{1} \\
0 \\
0 \\
0\end{array}$ & 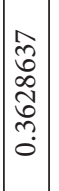 & $\mid$\begin{tabular}{c|}
$\overrightarrow{0}$ \\
0 \\
0 \\
0 \\
0 \\
0 \\
0
\end{tabular} & $\left|\begin{array}{l}1 \\
\infty \\
0 \\
0 \\
0 \\
0 \\
0 \\
0 \\
0\end{array}\right|$ & 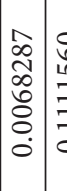 & $\begin{array}{l}8 \\
: \\
\stackrel{0}{0} \\
\Xi \\
\vdots \\
0\end{array}$ & 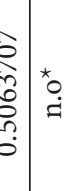 & 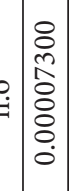 & 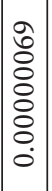 & 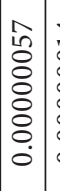 & 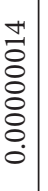 \\
\hline 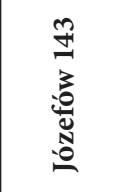 & $\begin{array}{l}\overrightarrow{6} \\
\vec{i} \\
\text { (n) }\end{array}$ & $\begin{array}{c}\text { Sू } \\
\text { in } \\
\text { in }\end{array}$ & $\stackrel{\overbrace{}}{\exists}$ & 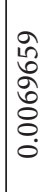 & I & $\begin{array}{l}\infty \\
0 \\
\infty \\
0 \\
0 \\
0 \\
0\end{array}$ & 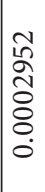 & $\mid \begin{array}{c}\tilde{m} \\
\tilde{\sigma} \\
\stackrel{g}{0} \\
0 \\
0 \\
0\end{array}$ & \begin{tabular}{|c|}
$\infty$ \\
2 \\
0 \\
$\hat{N}$ \\
0 \\
$\tilde{\infty}$ \\
0 \\
0
\end{tabular} & $\mid \begin{array}{c}\tilde{n} \\
\tilde{D} \\
0 \\
\delta \\
0 \\
0 \\
0\end{array}$ & $\left|\begin{array}{l}2 \\
\hat{\widehat{o}} \\
0 \\
0 \\
0 \\
0\end{array}\right|$ & 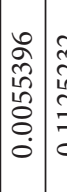 & 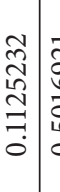 & 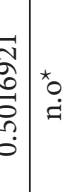 & 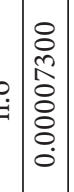 & $\begin{array}{l}0 \\
\dot{0} \\
0 \\
0 \\
0 \\
0 \\
0 \\
0 \\
0\end{array}$ & 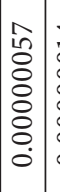 & \begin{tabular}{l}
\multirow{1}{*}{} \\
\\
0 \\
0 \\
$\vdots$ \\
$\vdots$ \\
0
\end{tabular} \\
\hline 莺 & $\begin{array}{l}\text { 芯 } \\
\text { i }\end{array}$ & $\begin{array}{l}\overrightarrow{0} \\
i \\
i\end{array}$ & $\begin{array}{c}\hat{n} \\
i \\
i\end{array}$ & $\begin{array}{l}\infty \\
\stackrel{2}{7} \\
\stackrel{7}{\Xi} \\
\vdots \\
0 \\
0\end{array}$ & । & 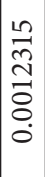 & 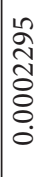 & 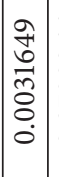 & $\mid \begin{array}{l}0 \\
0 \\
0 \\
2 \\
2 \\
\hat{0} \\
0 \\
0\end{array}$ & 11 & 1 & 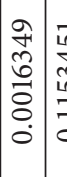 & $\begin{array}{c}\vec{n} \\
\stackrel{5}{+} \\
\stackrel{n}{n} \\
\vdots \\
0 \\
0\end{array}$ & 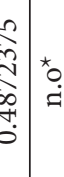 & 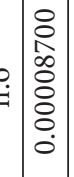 & 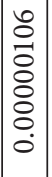 & \begin{tabular}{|l|}
+ \\
0 \\
0 \\
0 \\
$\vdots$ \\
$\vdots$ \\
$\vdots$ \\
$\vdots$ \\
0 \\
0
\end{tabular} & $\begin{array}{l}\infty \\
\dot{0} \\
0 \\
0 \\
\vdots \\
0 \\
\dot{0}\end{array}$ \\
\hline 葋 & $\begin{array}{l}\hat{\widehat{O}} \\
\underset{i}{i}\end{array}$ & $\begin{array}{l}\widehat{\widehat{G}} \\
\text { i. }\end{array}$ & $\mid \begin{array}{l}\stackrel{\hat{i}}{\mathrm{i}} \\
\mid\end{array}$ & 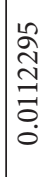 & । & 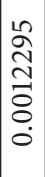 & $\begin{array}{l}\overrightarrow{\widehat{T}} \\
\text { ปे } \\
\vdots \\
0 \\
0\end{array}$ & 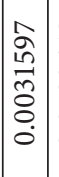 & 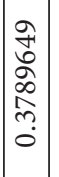 & 11 & 1 & 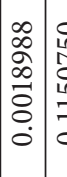 & 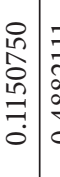 & 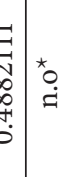 & 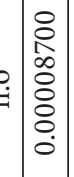 & \begin{tabular}{|l|}
0 \\
0 \\
0 \\
0 \\
0 \\
0 \\
0 \\
0 \\
0
\end{tabular} & \begin{tabular}{|l|} 
\\
0 \\
0 \\
0 \\
$\vdots$ \\
$\vdots$ \\
$\vdots$ \\
0 \\
0
\end{tabular} & $\begin{array}{l}\infty \\
0 \\
0 \\
0 \\
0 \\
0 \\
\dot{0}\end{array}$ \\
\hline 党 & 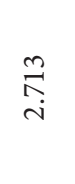 & $\underset{i}{\vec{i}}$ & $\stackrel{7}{3}$ & 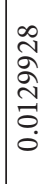 & I & \begin{tabular}{l}
0 \\
0 \\
\multirow{2}{1}{} \\
0 \\
0 \\
0 \\
0
\end{tabular} & 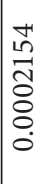 & $\mid \begin{array}{l}0 \\
0 \\
⿱ 亠 1 \\
+ \\
0 \\
0 \\
0 \\
0\end{array}$ & $\left|\begin{array}{c}\infty \\
2 \\
\right.$\cline { 1 - 1 } \\
\\
0 \\
0 \\
0\end{array}$|$ & $\mid \begin{array}{l}\infty \\
0 \\
0 \\
0 \\
0 \\
0 \\
0 \\
0\end{array}$ & 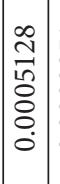 & 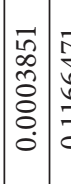 & 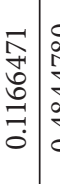 & 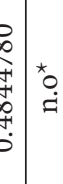 & 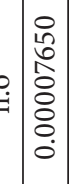 & 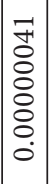 & 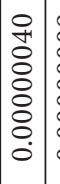 & $\begin{array}{l}2 \\
\dot{0} \\
\dot{0} \\
\dot{0} \\
\dot{0} \\
\dot{0}\end{array}$ \\
\hline 焉 & $\underset{i}{\stackrel{i}{i}}$ & 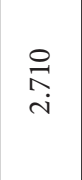 & $\stackrel{9}{0}$ & $\begin{array}{l}\hat{\widehat{J}} \\
0 \\
0 \\
0 \\
0 \\
0\end{array}$ & । & 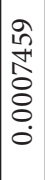 & 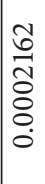 & $\mid \begin{array}{l}n \\
\hat{1} \\
b \\
+ \\
0 \\
0 \\
0 \\
0\end{array}$ & 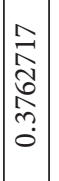 & $\mid \begin{array}{l}\infty \\
\tilde{0} \\
0 \\
0 \\
0 \\
0 \\
0 \\
0\end{array}$ & $\left|\begin{array}{l}2 \\
y \\
\forall \\
0 \\
0 \\
0 \\
0 \\
0\end{array}\right|$ & 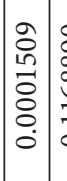 & 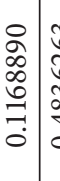 & 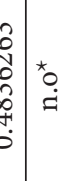 & 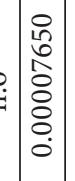 & 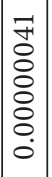 & $\left|\begin{array}{c}0 \\
+ \\
0 \\
0 \\
0 \\
\vdots \\
\vdots \\
0 \\
0 \\
0\end{array}\right|$ & $\begin{array}{l}\hat{0} \\
\dot{0} \\
\vdots \\
\vdots \\
0 \\
\dot{0}\end{array}$ \\
\hline 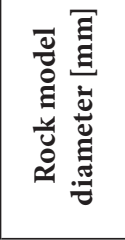 & 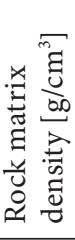 & 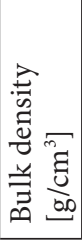 & & & & 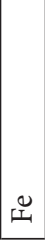 & $\Sigma$ & $|\Sigma|$ & $\tilde{0}$ & $\ddot{z}$ & \pm 1 & I & u & ه| & $\vec{U}$ & $\overrightarrow{0}$ & हี & 회 \\
\hline
\end{tabular}




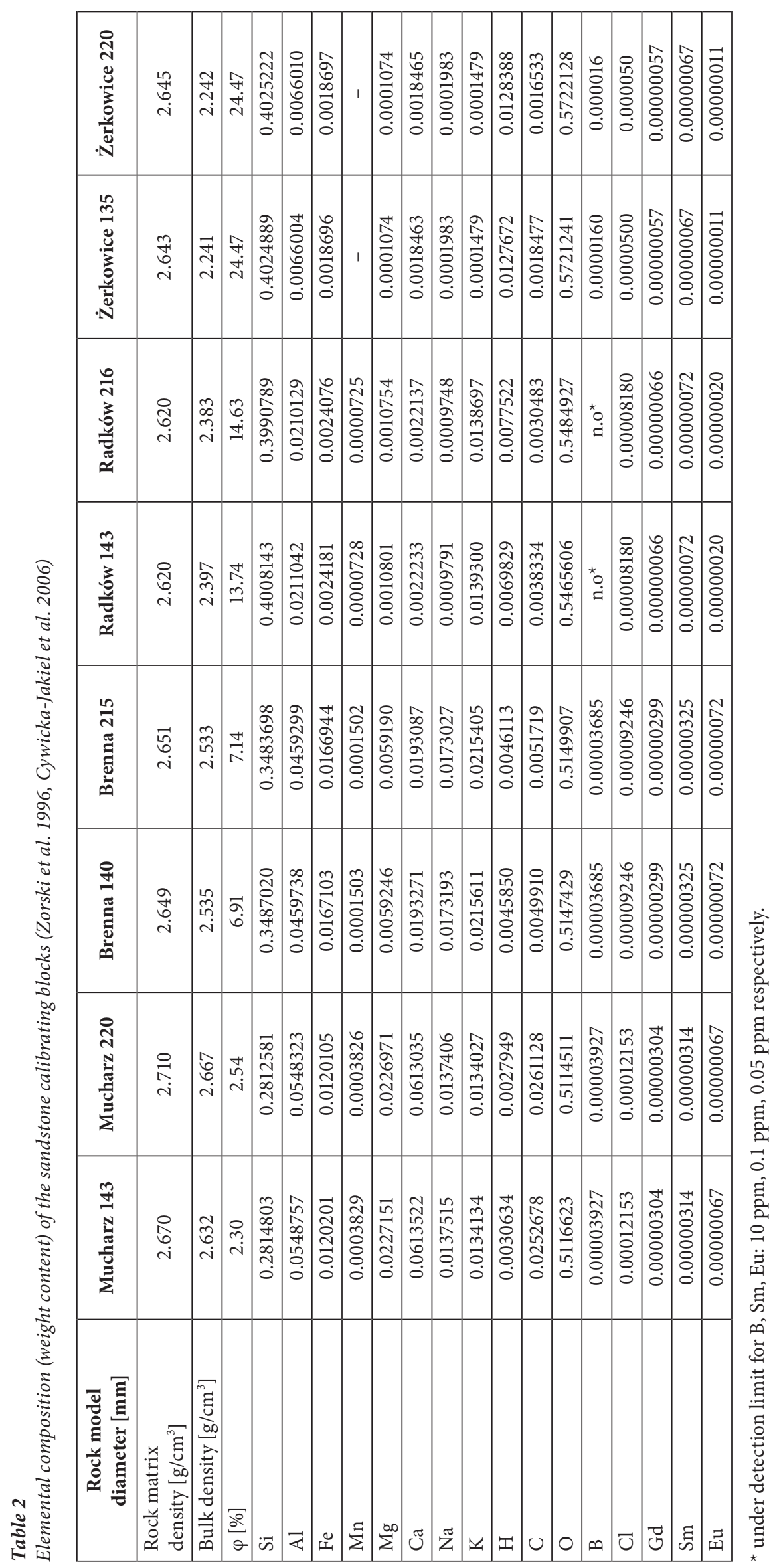




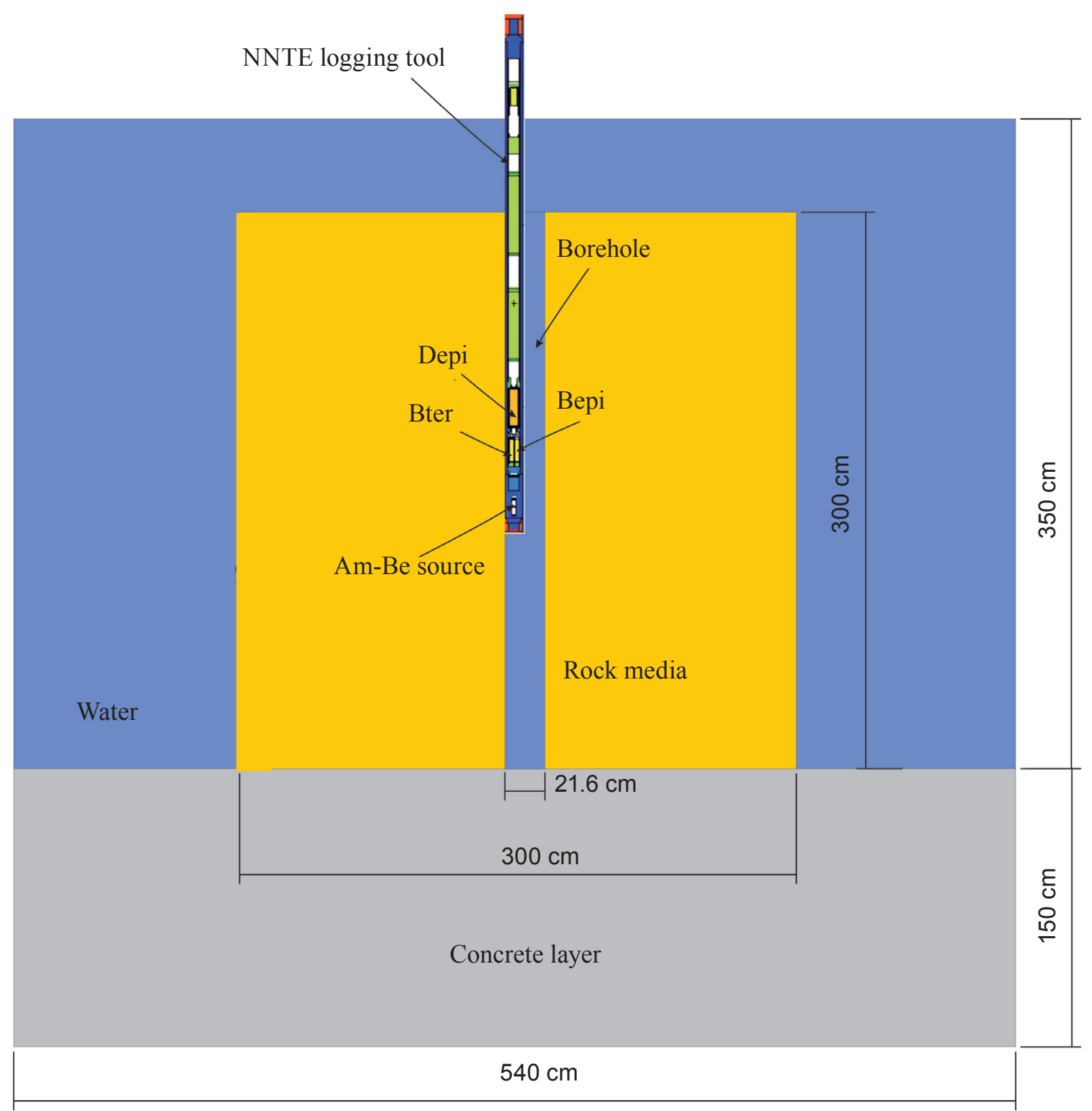

Fig. 2. Measurement geometry and position of the NNTE probe inside the calibration block

The correlation between the numerically calculated detector responses and the experimental results were not satisfactorily correlated for the Bter detector (Fig. $3 \mathrm{~A}, R^{2}=0.93$ ). The correlation coefficients for other detectors were better: for Bepi was $R^{2}=0.99$ and for Depi was $R^{2}=0.98$. The correlation coefficient has a lower value in relation to the dependence between the response of the Bter detector on the thermal neutron absorption cross section, $\Sigma_{a}$, of the medium. This suggestion was confirmed when a slight admixture of strong neutron absorbers (boron and rare earths) in the elemental composition of calibration blocks were included in the MCNP calculations. The detailed elemental analyses were performed (Cywicka-Jakiel et al. 2006) in the XRAL Laboratory (Canada) and the presence of the boron and rare earths in the rock models confirmed (Tab. 2).

The correlation coefficient for the Bter detector has been improved to $R^{2}=0.97$ (Fig. 3B) when these data have been taken into MCNP calculations. 
$\mathrm{A}$

MCNP calculations vs. measurements.

Basic rock chemical composition without admixture of neutron high absorbers.

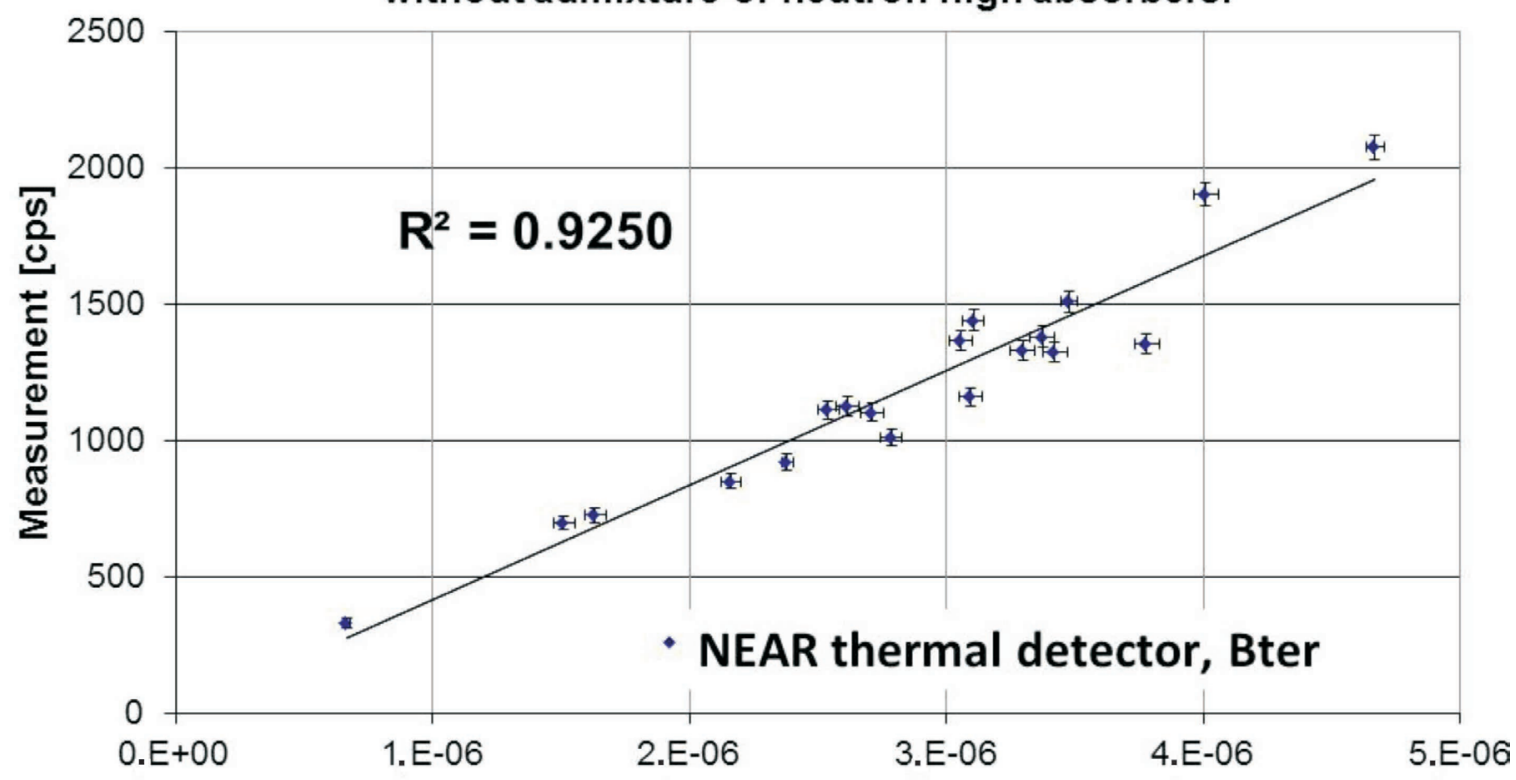

Numerical results [number of counts per source neutron]

$\mathrm{B}$

MCNP calculations vs. measurements.

Rock chemical composition together

with admixture of neutron high absorbers (XRAL analysis).

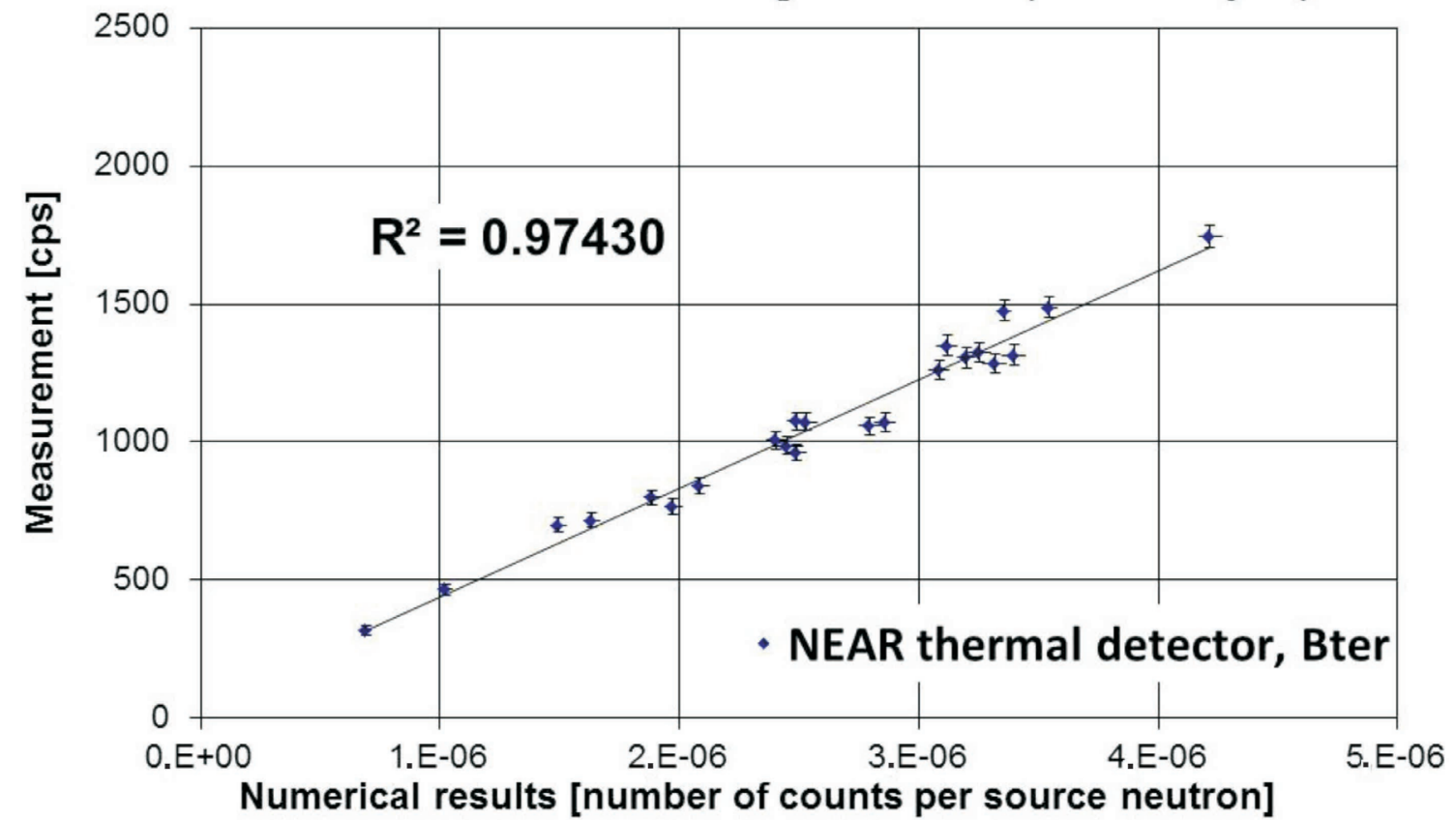

Fig. 3. Correlation between numerically calculated "near" thermal detector responses (MCNP) and measurements performed in the calibration blocks of the Zielona Góra facility: A) the basic elemental composition (weight content) of the calibrating blocks is taken as input data to the MCNP calculation (Tabs. 1, 2), (Drabina et al. 2003); B) the contribution of highly absorbing elements in Zielona Góra lithology models is taken into account (Tab. 3) 
The total amount of boron, chlorine and rare earths in the elemental composition of calibration blocks at Zielona Góra facility does not extend beyond $0.02 \%$ of weight content (e.g. Mucharz 143, Fig. 4). All of these constituents have a strong influence on the neutron parameters of the media. Boron is present in sandstone blocks, while in limestones and dolomites only chlorine is to be found. To present the effect of the $\mathrm{B}, \mathrm{Cl}$ and rare earths admixture in calibration blocks, the thermal neutron absorption cross section has been calculated twice with and without neutron absorbers content. The results are presented in Figure 5 and Table 3.

\section{Table 3}

The thermal neutron absorption cross section calculated for standard calibration blocks at Zielona Góra facility for the main elemental compositions $\left(\Sigma_{a 1}\right)$ and taking into account the small admixtures of B, Cl, Gd, Sm, Eu absorbers $\left(\Sigma_{a 2}\right)$. Linear macroscopic cross section $\Sigma_{a}$ is calculated for the neutron speed $v=2200 \mathrm{~ms}^{-1}$ and is expressed in capture units (c.u.), where $1 \mathrm{c.u}=10^{3} \mathrm{~cm}^{-1}$

\begin{tabular}{|c|c|c|c|c|}
\hline $\begin{array}{l}\text { Rock model diameter } \\
{[\mathrm{mm}]}\end{array}$ & $\begin{array}{c}\text { Rock matrix density } \\
{\left[\mathrm{g} / \mathrm{cm}^{3}\right]}\end{array}$ & $\begin{array}{c}\Sigma_{a 1} \\
\sigma\left(\Sigma_{a 1}\right) \\
\text { [c.u.] }\end{array}$ & $\begin{array}{c}\Sigma_{a 2} \\
\sigma\left(\Sigma_{a 2}\right) \\
\text { [c.u.] }\end{array}$ & [\%] \\
\hline Biała Marianna 141 & 2.712 & $\begin{array}{l}7.09 \\
0.03\end{array}$ & $\begin{array}{l}8.50 \\
0.03\end{array}$ & 16.66 \\
\hline Biała Marianna 220 & 2.713 & $\begin{array}{l}7.21 \\
0.03\end{array}$ & $\begin{array}{l}8.62 \\
0.03\end{array}$ & 16.35 \\
\hline Morawica 141 & 2.677 & $\begin{array}{l}7.93 \\
0.03\end{array}$ & $\begin{array}{l}9.80 \\
0.03\end{array}$ & 19.08 \\
\hline Morawica 220 & 2.674 & $\begin{array}{l}7.80 \\
0.03\end{array}$ & $\begin{array}{l}9.66 \\
0.03\end{array}$ & 19.25 \\
\hline Józefów 143 & 2.691 & $\begin{array}{l}9.69 \\
0.03\end{array}$ & $\begin{array}{c}11.34 \\
0.03\end{array}$ & 14.55 \\
\hline Józefów 216 & 2.686 & $\begin{array}{c}10.28 \\
0.03\end{array}$ & $\begin{array}{c}11.93 \\
0.03\end{array}$ & 13.83 \\
\hline Pińczów 145 & 2.716 & $\begin{array}{c}16.60 \\
0.03\end{array}$ & $\begin{array}{c}18.17 \\
0.03\end{array}$ & 8,64 \\
\hline Pinczów 220 & 2.694 & $\begin{array}{c}15.90 \\
0.03\end{array}$ & $\begin{array}{c}17.45 \\
0.03\end{array}$ & 8.88 \\
\hline Libiąż 145 & 2.823 & $\begin{array}{l}7.48 \\
0.02\end{array}$ & $\begin{array}{l}9.33 \\
0.02\end{array}$ & 19.82 \\
\hline Libiąż 216 & 2.824 & $\begin{array}{l}7.94 \\
0.02\end{array}$ & $\begin{array}{l}9.79 \\
0.02\end{array}$ & 18.90 \\
\hline Mucharz 143 & 2.670 & $\begin{array}{l}9.01 \\
0.01\end{array}$ & $\begin{array}{c}15.42 \\
0.01\end{array}$ & 42.68 \\
\hline Mucharz 220 & 2.710 & $\begin{array}{l}9.00 \\
0.01\end{array}$ & $\begin{array}{c}15.96 \\
0.01\end{array}$ & 43.61 \\
\hline Brenna 140 & 2.649 & $\begin{array}{c}10.56 \\
0.01\end{array}$ & $\begin{array}{c}16.58 \\
0.01\end{array}$ & 36.31 \\
\hline Brenna 215 & 2.651 & $\begin{array}{c}10.57 \\
0.01\end{array}$ & $\begin{array}{c}16.60 \\
0.01\end{array}$ & 36.33 \\
\hline Radków 143 & 2.620 & $\begin{array}{l}9.24 \\
0.01\end{array}$ & $\begin{array}{c}10.86 \\
0.01\end{array}$ & 14.92 \\
\hline Radków 216 & 2.620 & $\begin{array}{l}9.62 \\
0.01\end{array}$ & $\begin{array}{c}11.24 \\
0.01\end{array}$ & 14.41 \\
\hline Żerkowice 135 & 2.643 & $\begin{array}{c}10.90 \\
0.01\end{array}$ & $\begin{array}{c}13.11 \\
0.01\end{array}$ & 16.86 \\
\hline Żerkowice 220 & 2.645 & $\begin{array}{c}10.94 \\
0.01\end{array}$ & $\begin{array}{c}13.15 \\
0.01\end{array}$ & 16.81 \\
\hline
\end{tabular}




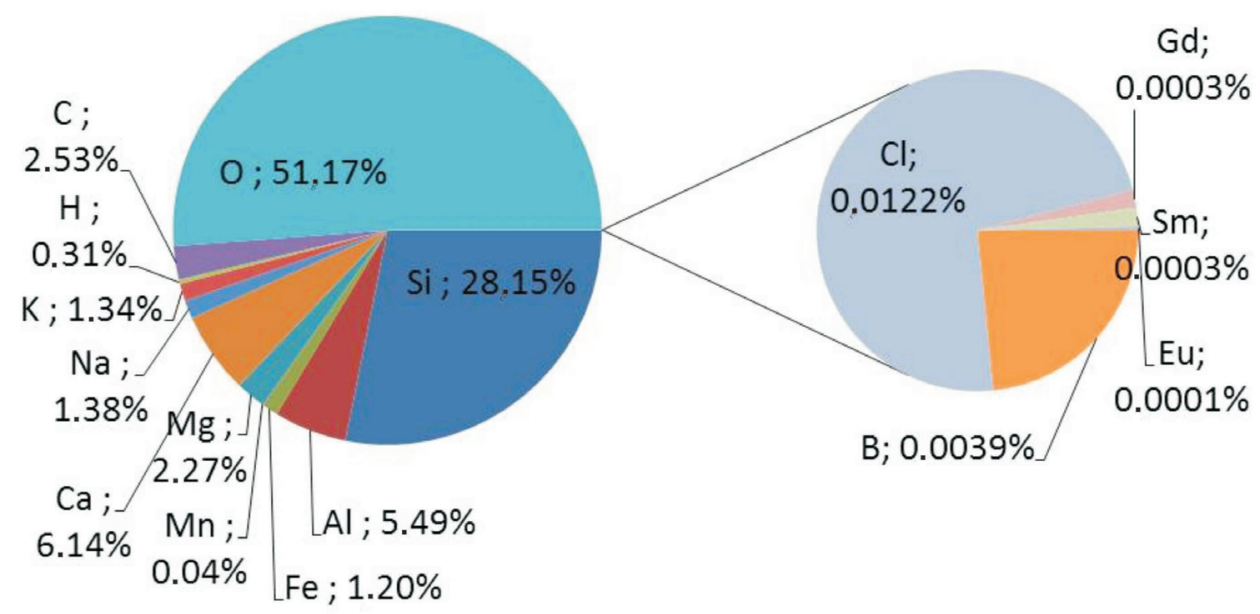

Fig. 4. Example of the elemental composition of the sandstone Mucharz 143 calibration block indicating the contents of strong neutron absorbers

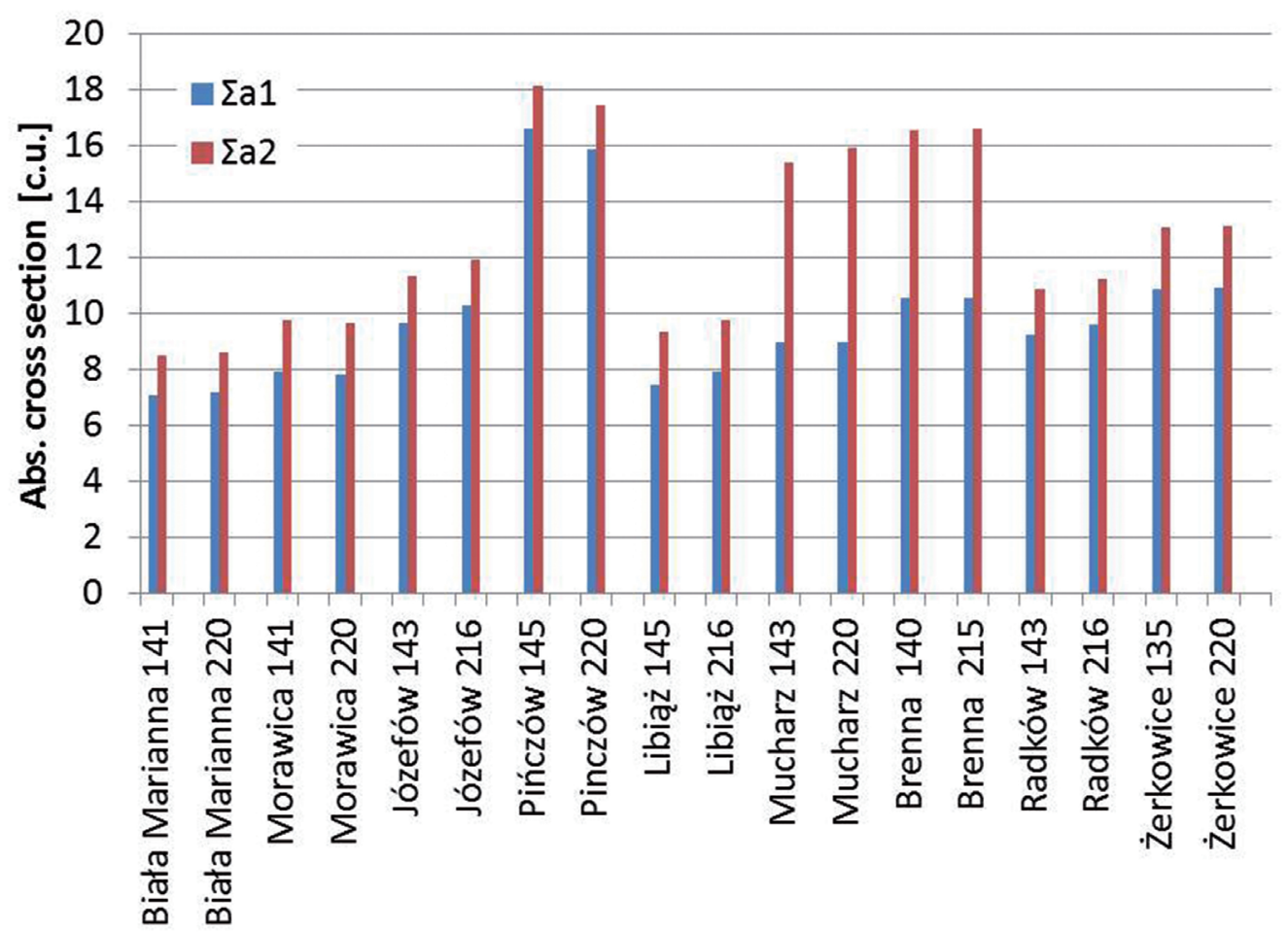

Fig. 5. Thermal neutron absorption cross section calculated for standard calibration blocks at Zielona Góra facility for the main elemental compositions $\left(\Sigma_{a 1}\right)$ and taking into account the small admixtures of $B, C l$ and rare earth absorbers $\left(\Sigma_{a 2}\right)$

The standard deviations $\sigma\left(\Sigma_{a 1}\right)$ and $\sigma\left(\Sigma_{a 2}\right)$ presented in Table 3 have been calculated taking into account only the standard deviations for particular elements based on Mughabghab (1984). The parameter $\Delta$ is a relative error of the $\Sigma_{a}$ in two cases: with and without taking into account the small admixtures of strong neutron absorbers: $\mathrm{B}, \mathrm{Cl}$ and rare earths. It was introduced into Table 3 to illustrate the influence of the small admixtures of strong neutron absorbers: $\mathrm{B}, \mathrm{Cl}$ and rare earths on the macroscopic neutron absorption cross section of given models. 
The very good correlation between the real experimental results obtained at the Zielona Góra facility and the results of the numerically reproduced experiments confirm that the problem has been modeled correctly. This compatibility allows us to realize reliable numerical experiments for other interesting measurements with neutron probes.

\section{MONTE CARLO CALIBRATION OF THE NNTE LOGGING TOOL FOR STANDARD MIOCENE LITHOLOGY}

In order to obtain a quantitative interpretation of the results of borehole measurements, the reading tool need to be calibrated. The aim of the calibration is to find a correlation between the detector response and the physical properties of rock. There are many parameters (side factors) which influence the correlation, e.g. borehole dimension, composition of borehole fluid, eccentric position of the probe. All of them should be taken into consideration during the calibration of the neutron logging tool. Calibration can be done experimentally by the real measurements of the tool response in the set of well-known geological standards. For the neutron tools, the semi-empirical calibration method has been developed by Prof. J.A. Czubek (Czubek 1988). The method is a combination of an analytical solution of the neutron transport in the borehole and the surrounding geological medium with some experiments performed on a calibration facility. Experimental calibration is the most costly, because it requires constructing appropriate physical models to analyze the influence of all side factors. It is possible to extend the results of the experimental calibration onto a wide range of parameters and side factors. Sometimes it is impossible to build a physical model of a rock of desired parameters. In such situations, the numerical methods are the solution.
Numerical Monte-Carlo methods offer another possibility of calibration and can be regarded as a parallel method to the experimental one. Monte Carlo methods create the possibility to build any facility system in a relatively easy way. To that end the numerical model of the whole calibration facility system must be modeled in detail. The response of the neutron tool can be calculated for any combination of parameters of the borehole and the rock medium.

The correctness of the simulation procedure, as described above in Section "MCNP Benchmark Calculation", allowed the creation of a set of standard calibration curves for the NNTE probe (Drabina \& Zorski 2005). The response of the each detector has been simulated for the lithology standard, i.e. for the Miocene lithology with the elemental composition and the density $\rho_{m a}=2.65 \mathrm{~g} \mathrm{~cm}^{-3}$ presented in Table 4 (Zorski \& Stadtmüller 2001).

The $\Sigma_{a}$ of the Miocene lithology according to the elemental composition given in Table 4 is equal to $7.75 \mathrm{c}$.u. It is possible to modify the $\Sigma_{a}$ by means of a very small admixture of a strong thermal neutron absorber like ${ }^{10} \mathrm{~B}$ without significant change of the weight content of the considered medium. For example admixture of the $0.0016318 \%$ of ${ }^{10} \mathrm{~B}$ enable to obtain the thermal neutron absorption cross section $\Sigma_{a}=15$ c.u. of the above Miocene standard. That possibility of modification of $\Sigma_{a}$ is commonly used for calculating corrections for calibration curves.

In order to obtain the calibration curves the response of each detector has been calculated as a function of porosity. The porosity was changed in the range from $0 \%$ to $100 \%$. Borehole diameter was $216 \mathrm{~mm}$ and filled with water and the NNTE tool was decentralized. All of the calibration curves presented in this paper were obtained for a value of $\Sigma_{a}=15$ c.u. (Fig. 6). The red crosses presented in Figure 6 are the extra control points added only for the validation of calculations, as described above in Section "MCNP Benchmark Calculation".

Table 4

Elemental composition of the standard Miocene lithology [\%]

\begin{tabular}{|c|c|c|c|c|c|c|}
\hline $\mathrm{SiO}_{2}$ & $\mathrm{Al}_{2} \mathrm{O}_{3}$ & $\mathrm{Fe}_{2} \mathrm{O}_{3}$ & $\mathrm{CaO}$ & $\mathrm{K}_{2} \mathbf{O}$ & $\mathrm{CO}_{2}$ & $\mathrm{H}_{2} \mathrm{O}$ \\
\hline 72.5 & 7 & 2 & 7.5 & 1.8 & 8 & 1.2 \\
\hline
\end{tabular}




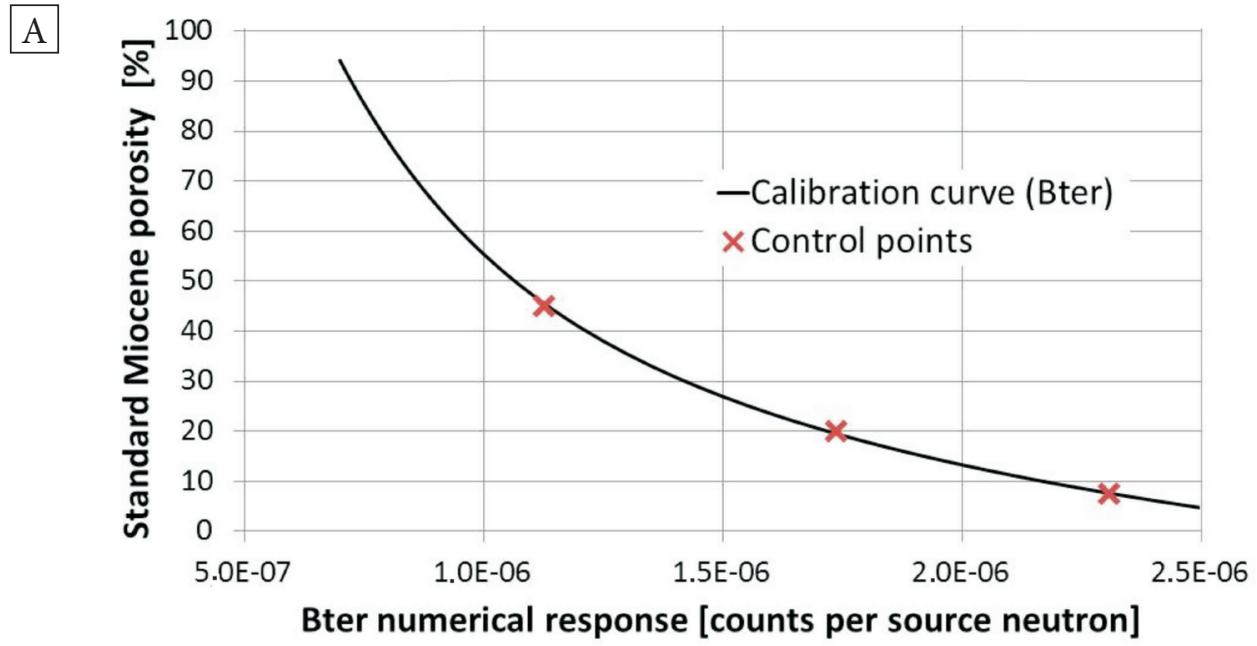

B

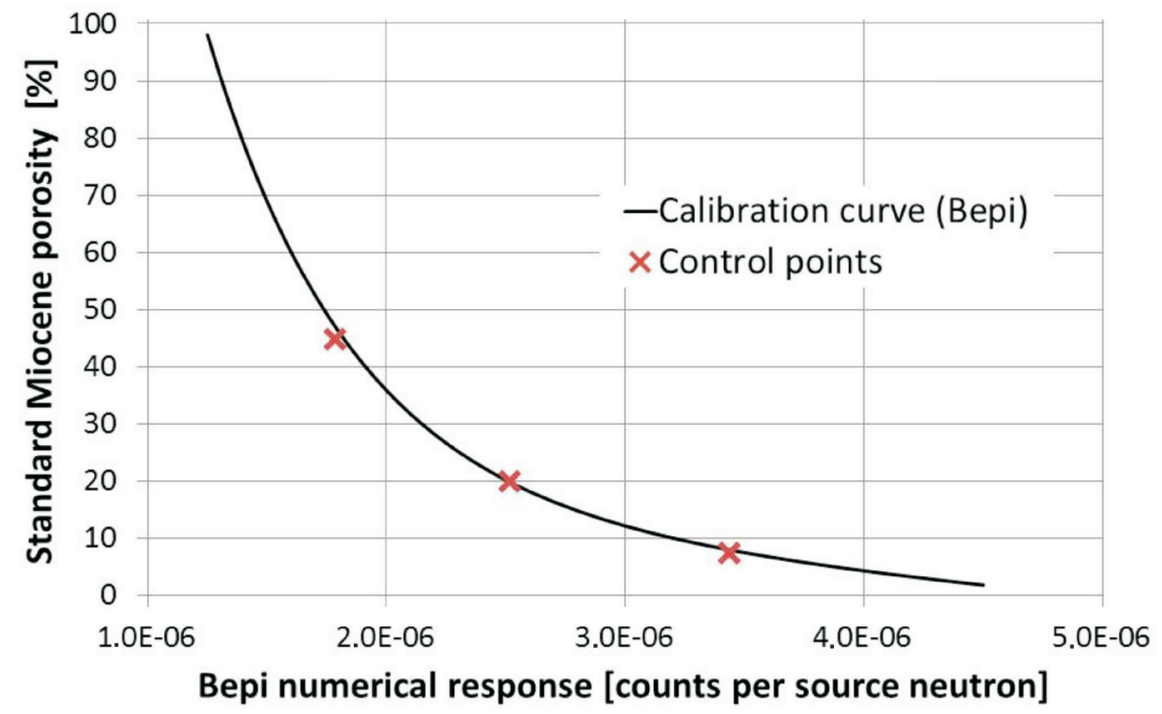

C

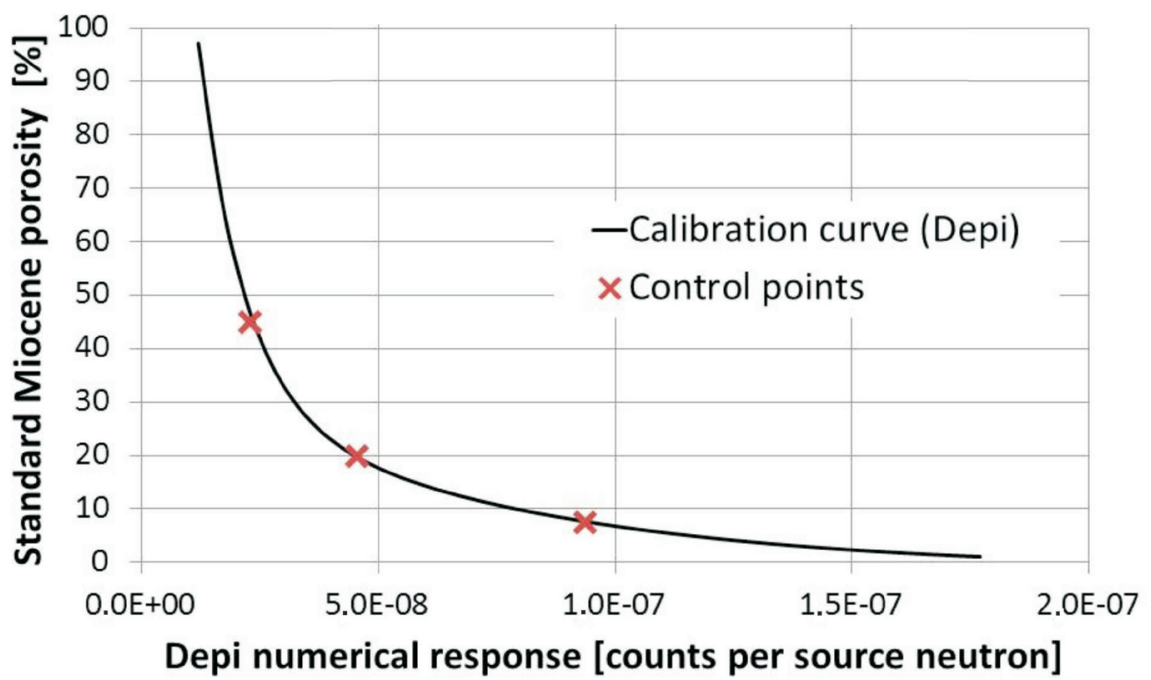

Fig. 6. Standard calibration curves calculated numerically by the MCNP for Miocene lithology for Bter, Bepi and Depi detectors of NNTE probe 
The best fit of the calculated points was obtained by using the sum of exponential curves. The final calibration curves are described by the following equation:

$$
y=y_{0}+A_{1} \exp \left(-\frac{x-x_{0}}{t_{1}}\right)+A_{2} \exp \left(-\frac{x-x_{0}}{t_{2}}\right),
$$

where $y$ is the porosity of the standard Miocene lithology [\%] and $x$ is the numerically calculated response of the detector: Bter, Bepi, Depi expressed in the number of counts in a given detector corresponding to one neutron escaping from the neutron source. The parameters of the above equation do not have a physical significance and for each detector are collected in Table 5.

Tab. 5. Parameters of calibration curves obtained from the MCNP calculations for three detectors of the NNTE tool

\begin{tabular}{|c|c|c|c|}
\cline { 2 - 4 } \multicolumn{1}{c|}{} & Bter & Bepi & Depi \\
\hline$y_{0}$ & -18.75332 & -117.83013 & -1.48304 \\
\hline$x_{0}$ & $5.7577 \cdot 10^{-7}$ & $1.2139 \cdot 10^{-6}$ & $8.9672 \cdot 10^{-9}$ \\
\hline$A_{1}$ & 67.4822 & 88.25646 & 91.19472 \\
\hline$A_{2}$ & 72.27604 & 133.11497 & 33.75238 \\
\hline$t_{1}$ & $3.202 \cdot 10^{-7}$ & $6.0607 \cdot 10^{-7}$ & $9.5803 \cdot 10^{-9}$ \\
\hline$t_{2}$ & $1.6988 \cdot 10^{-6}$ & $3 \cdot 10^{-5}$ & $6.4658 \cdot 10^{-8}$ \\
\hline
\end{tabular}

\section{NUMERICAL IMAGING OF THE NNTE TOOL RESPONSE IN STANDARD MIOCENE LITHOLOGY}

One of the important problems of the interpretation of neutron detectors signals is the spatial range of the neutron probe. The average distance that a neutron can travel in a given bulk medium during the slowing down and diffusion processes are: $\left\langle r_{s}\right\rangle=\sqrt{6} \cdot L_{s}$ and $\left\langle r_{d}\right\rangle=\sqrt{6} \cdot L_{d}$, respectively; where $L_{s}$ is a neutron slowing-down length, and $L_{d}$ is a neutron diffusion length. The total migration length $L_{m}$ can be calculated from the formula: $L_{m}^{2}=L_{s}^{2}+L_{d}^{2}$, and constantly: $\left\langle r_{m}\right\rangle=\sqrt{6} \cdot L_{m}$. Knowing these parameters, the radial range of neutron migration in the medium can be estimated. E.g. for water $L_{s}$ is about $7 \mathrm{~cm}$ and $L_{d}$ about $2.8 \mathrm{~cm}$. For comparison: for $\mathrm{SiO}_{2} L_{s}$ is about $29 \mathrm{~cm}$ and $L_{d}$ about $19 \mathrm{~cm}$. The image of neutron penetration from the point neutron source in the isotropic, homogeneous medium is a sphere. Some examples for $\mathrm{SiO}_{2}$ and for water are presented in Figure 7. The distribution of the thermal neutrons in a cube of the side $50 \mathrm{~cm}$ from the point Am-Be neutron source located in the center has been calculated numerically.

The analogical visualization can be presented when the silica cube is cut by a hole filled with strongly absorbing brine. The example presented in Figure 8 has been calculated for the hole of $22 \mathrm{~cm}$ diameter filled by brine of $200 \mathrm{kppm}$ of $\mathrm{NaCl}$. The effect of the absorption of thermal neutrons by chlorine is visible (Fig. 8A). Additionally the unsymmetrical distribution of the thermal neutron field is observed when the neutron source is placed on the borehole wall (Fig. 8B).

The presented visualization of the neutron distribution were driven by an impulse to think of how the distribution of neutrons in more realistic conditions appears, what happens when a real probe is inside the borehole? We decided to show the distribution only these neutrons which reached the detectors of the probe meaning that we keep track of those neutrons which are finally absorbed in the detector. Neutrons which are absorbed in the depths of rock do not participate in the count of the detector (Woźnicka et al. 2012).The following numerical experiments have been carried out in order to show the distribution of neutrons referred above.

The response of each detector of the NNTE logging tool are calculated for the infinite homogenous standard Miocene lithology. Three cases are taken into account (Tab. 6): Miocene lithology of low porosity (7.5\%) and two different values of thermal neutron absorption (S_7.5/15 and S_7.5/40) and the third case: lithology of high porosity and high neutron absorption (S_45/40). The elemental composition of the standard Miocene lithology is taken as in Table 4. The rock pore spaces are saturated by water. The probe is decentralized and adjacent to the wall of the water filled borehole with diameter of $216 \mathrm{~mm}$. The external size of the simulated model is $10 \mathrm{~m} \times 10 \mathrm{~m} \times 10 \mathrm{~m}$. The geometric size of the rock model is selected so that all of the neutrons emitted from the source are absorbed therein. None of the neutrons are scattered outside simulated volume and this assumption means the simulated volume may be treated as an infinite medium in terms of the transport of neutrons from an Am-Be source. 

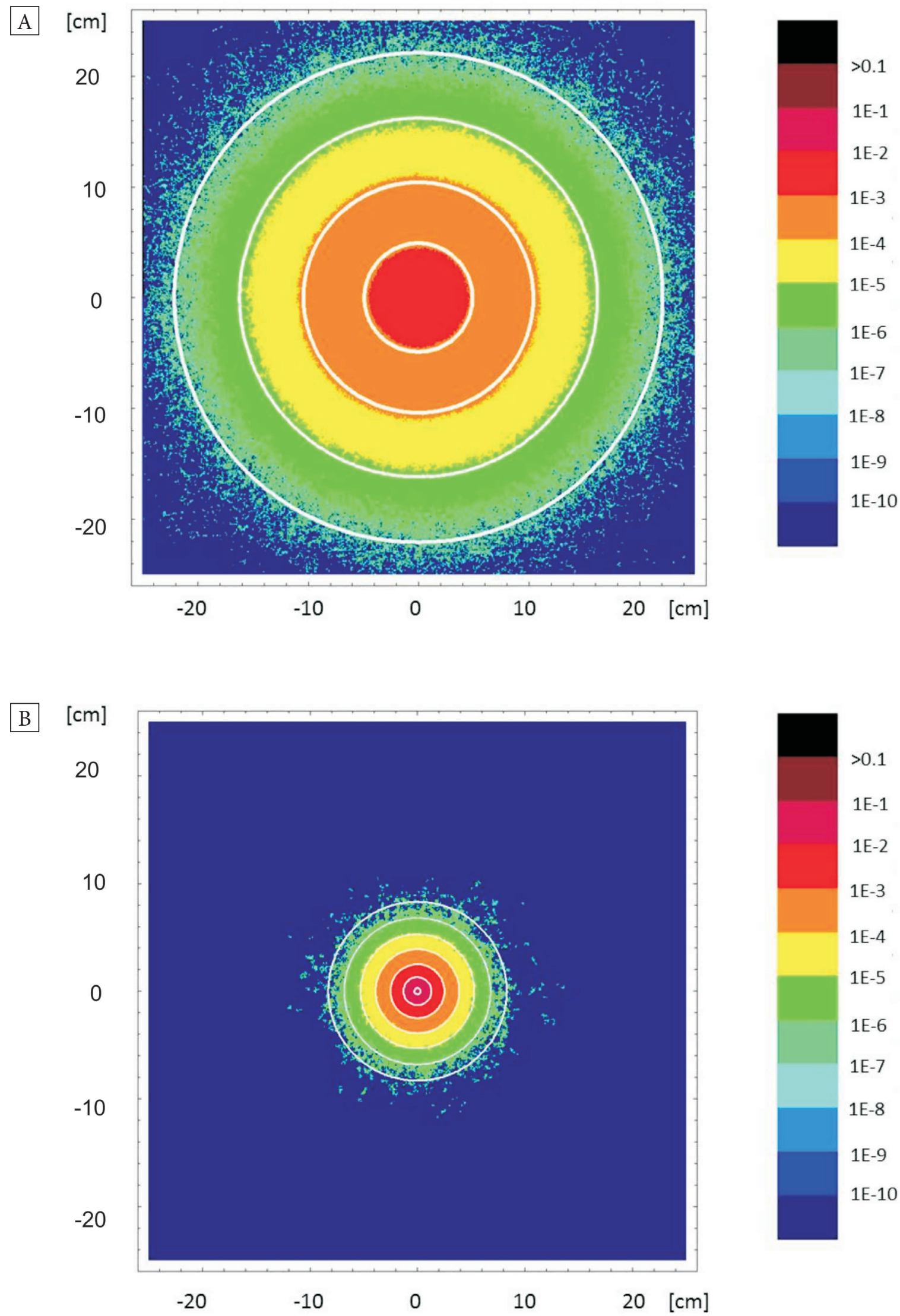

Fig. 7. Distribution of the thermal neutrons in a cube of side $50 \mathrm{~cm}$ from the point Am-Be neutron source located in the center: A) in silica; B) in water 

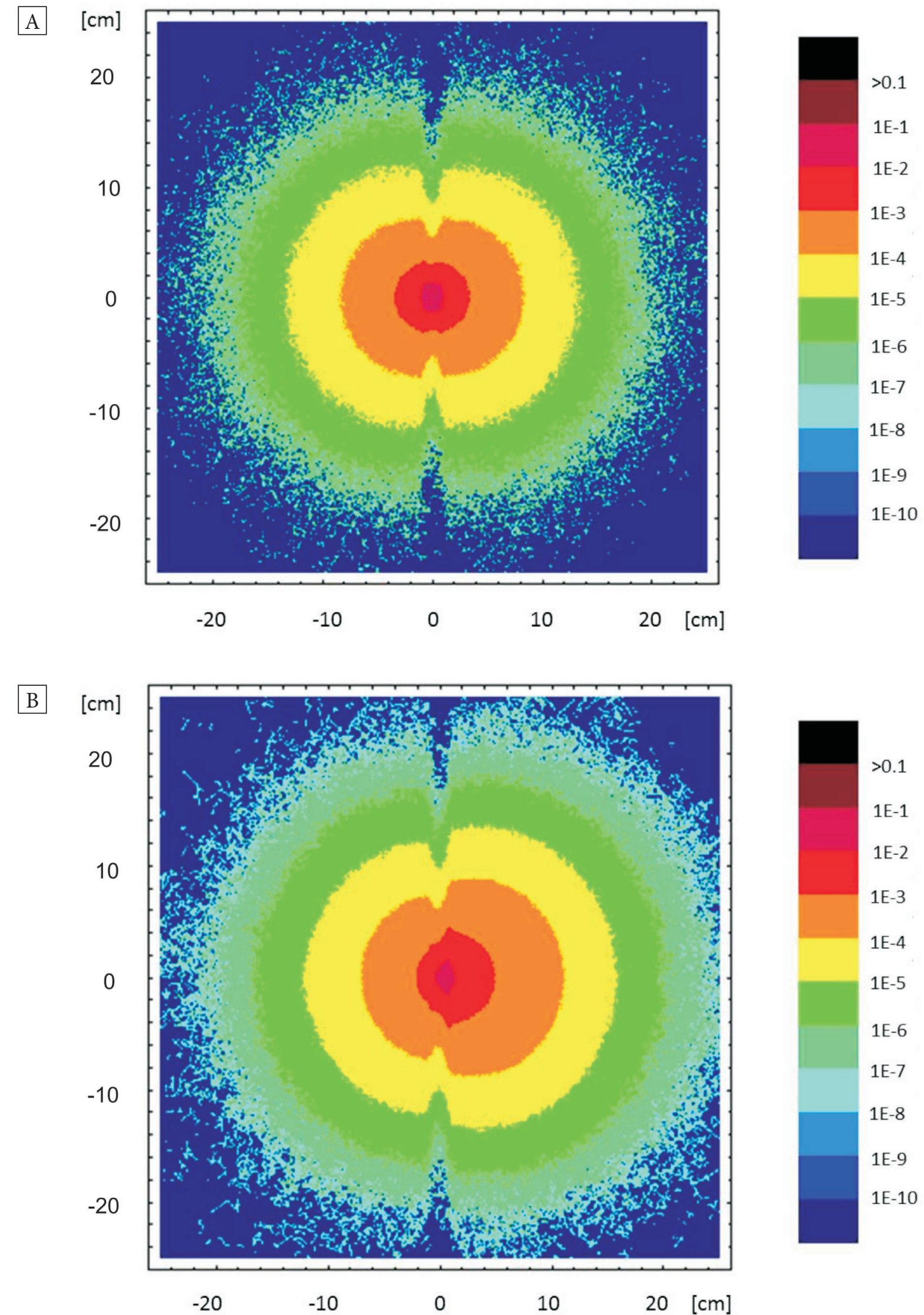

Fig. 8. Distribution of the thermal neutrons in the silica cube of side $50 \mathrm{~cm}$ cut by the hole of $22 \mathrm{~cm}$ diameter filled with $220 \mathrm{kppm}$ of $\mathrm{NaCl}$ brine from the point $\mathrm{Am}$-Be neutron source: $\mathrm{A})$ source on the axis of the hole; $B$ ) source on the wall of the hole 
To validate the model prepared for the MCNP simulations, the response of each detector corresponding to the three control points of the calibration curves have been calculated as shown in Figure 6. Control points are the detectors responses for standard Miocene lithology of 7.5\%,20\% and $40 \%$ of porosity and $\Sigma_{a}=15$ c.u. (Tab. 7, Fig. 9). In Figure 6 they are marked at the curves as red crosses. The full compliance of the results has been achieved.

In order to obtain a good statistical accuracy of calculations, the $1.5 \cdot 10^{8}$ histories of neutrons starting from the Am-Be source were simulated for each run. The relative errors below 3\% have been obtained. The response of a detector is defined as an average number of neutrons absorbed in a detector volume unit, per one starting particle from the source. The response of each detector is strongly related with parameters of the medium: the porosity $\varphi$ and the thermal neutron absorption cross section $\Sigma_{a}$. We assume detectors, which count, thermal (Bter) and epithermal (Bepi, Depi) neutrons with $100 \%$ efficiency.

The images of neutron 2D distribution in the outside borehole were drawn up for each of the presented models. This kind of chart (map) shows the origin of the neutrons registered in the detectors. A special MCNP technique called 'flagging' was used, which enables the calculation of the flux of particles that moved through selected cells of the model. In these calculations the source cell was flagged and the isolines of the map show the areas from which the same number of neutrons are counted (absorbed) in the detector. The color scale (here: from blue to red) is a measure of the growing number of neutrons reaching the detector from a given element of the space.

Based on these maps, the space range of the NNTE tool can be visualized. The examples of maps are presented for detector Bter in Figure 10 for detector Bepi in Figure 11 and for detector Depi in Figure 12.

In the presented maps the positon of the particular elements of the logging tool are the following: for the Am-Be neutron source $z=2 \mathrm{~cm}$, for the center of the near detectors: Bter and Bepi $z=31 \mathrm{~cm}$ and $x=0 \mathrm{~cm}$, and for the center of the far detector Depi $z=54.5 \mathrm{~cm}$ and $x=0 \mathrm{~cm}$. All of these elements are localized along borehole-media.

In order to be counted in the "near" thermal detector (Bter) the neutrons emitted from the Am-Be source must be slowing down and thermalized in the model considered. Fewer numbers of thermal neutrons are measured by the Bter when the absorption of the thermal neutron of the model is increased. These differences are shown in Figure 10A and Figure 10B. In both cases the space range of detector Bter is the same but the number of counts for case S_7.5/40 is lower on $23 \%$ in relation to the case S_7.5/40 (Tab. 7, Fig. 9).

Table 6

Porosity $\varphi$ and $\Sigma_{a}$ for Miocene models. $\Sigma_{\text {a matrix }}$ and $\Sigma_{\text {a rock }}$ - thermal neutron absorption cross section for rock matrix and rock model with water saturated pores

\begin{tabular}{|c|c|c|c|c|}
\hline Rock model & $\begin{array}{c}\varphi \\
{[\%]}\end{array}$ & $\begin{array}{c}\Sigma_{a \text { matrix }} \\
{[\text { c.u.] }}\end{array}$ & $\begin{array}{c}\Sigma_{a \text { rock }}{ }^{*} \\
\text { [c.u.] }\end{array}$ & $\begin{array}{c}\rho_{\mathrm{vol}} \\
{\left[\mathrm{g} / \mathrm{cm}^{3}\right]}\end{array}$ \\
\hline S_7.5/15 & 7.5 & 15 & 15.54 & 2.508 \\
\hline S_7.5/40 & 7.5 & 40 & 38.67 & 2.508 \\
\hline S_45/40 & 45.0 & 40 & 32.01 & 1.897 \\
\hline
\end{tabular}

$* \Sigma_{a \text { rock }}=(1-\varphi) \Sigma_{a \text { matrix }}+\varphi \Sigma_{a \text { water }} ; \Sigma_{a \text { water }}=22.24$ c.u.

Table 7

The response of the detectors of NNTE logging tool in infinity, homogenous rock Miocene models

\begin{tabular}{|c|c|c|c|c|c|c|}
\hline Rock model & $\begin{array}{c}\text { Bter } \\
{\left[\mathbf{a b s} / \mathbf{c m}^{3} / \mathbf{s n}\right]}\end{array}$ & $\begin{array}{c}\text { Relative error } \\
\boldsymbol{\sigma B t e r}[\%]\end{array}$ & $\begin{array}{c}\text { Bepi } \\
{\left[\mathbf{a b s} / \mathbf{c m}^{\mathbf{3}} \mathbf{s n}\right]}\end{array}$ & $\begin{array}{c}\text { Relative error } \\
\boldsymbol{\sigma B e p i}[\%]\end{array}$ & $\begin{array}{c}\text { Depi } \\
{\left[\mathbf{a b s} / \mathbf{c m}^{3} / \mathbf{s n}\right]}\end{array}$ & $\begin{array}{c}\text { Relative error } \\
\boldsymbol{\sigma D e p i}[\%]\end{array}$ \\
\hline S_7.5/15 & $2.31 \mathrm{E}-06$ & 0.53 & $3.44 \mathrm{E}-06$ & 0.60 & $9.40 \mathrm{E}-08$ & 1.23 \\
\hline S_7.5/40 & $1.79 \mathrm{E}-06$ & 0.57 & $3.42 \mathrm{E}-06$ & 0.60 & $9.08 \mathrm{E}-08$ & 1.26 \\
\hline S_45/40 & $9.08 \mathrm{E}-07$ & 0.84 & $1.79 \mathrm{E}-06$ & 0.83 & $2.27 \mathrm{E}-08$ & 2.57 \\
\hline
\end{tabular}




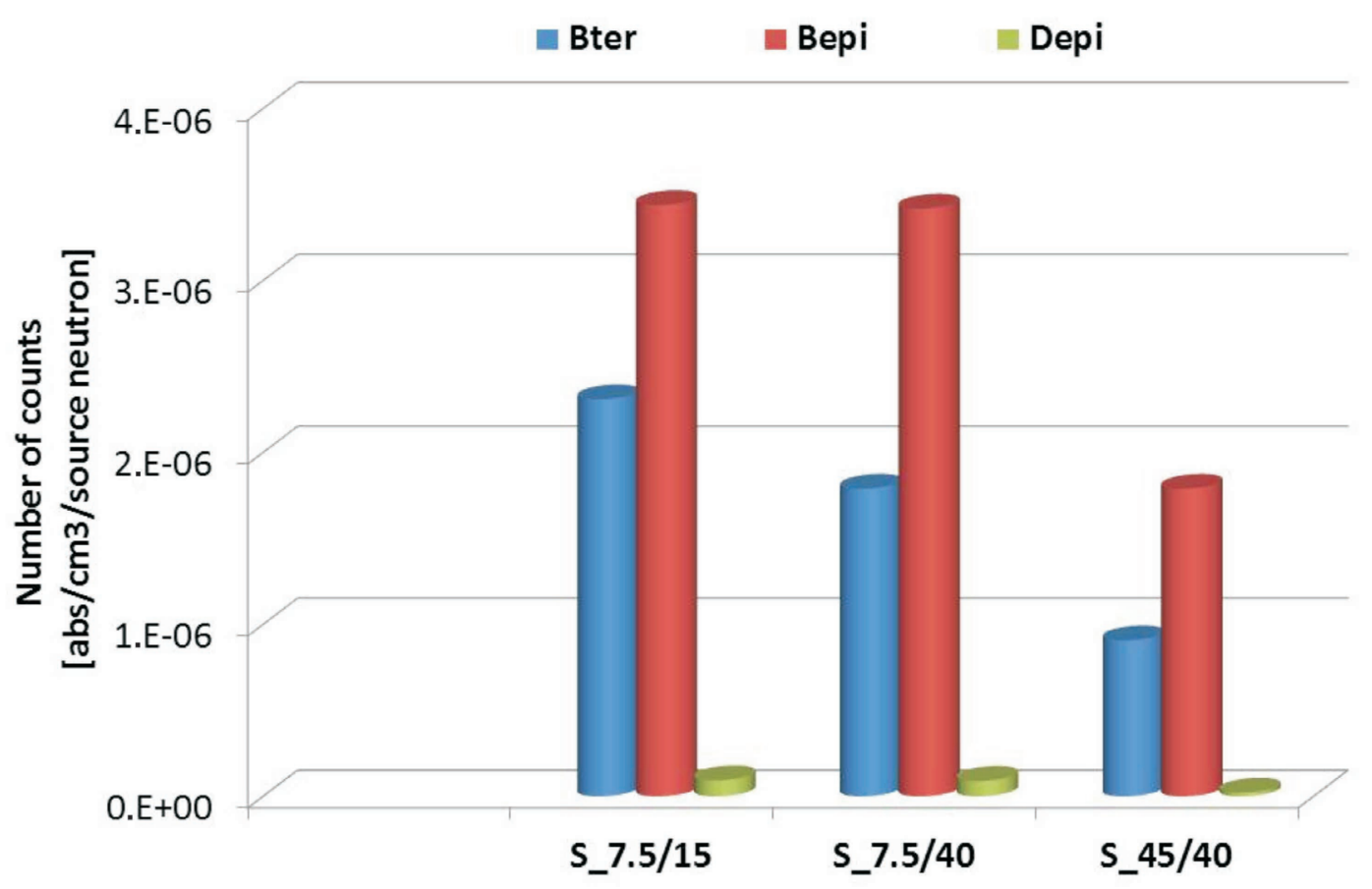

Fig. 9. Comparison of the number of counts of detectors Bter, Bepi and Depi for the three considered cases

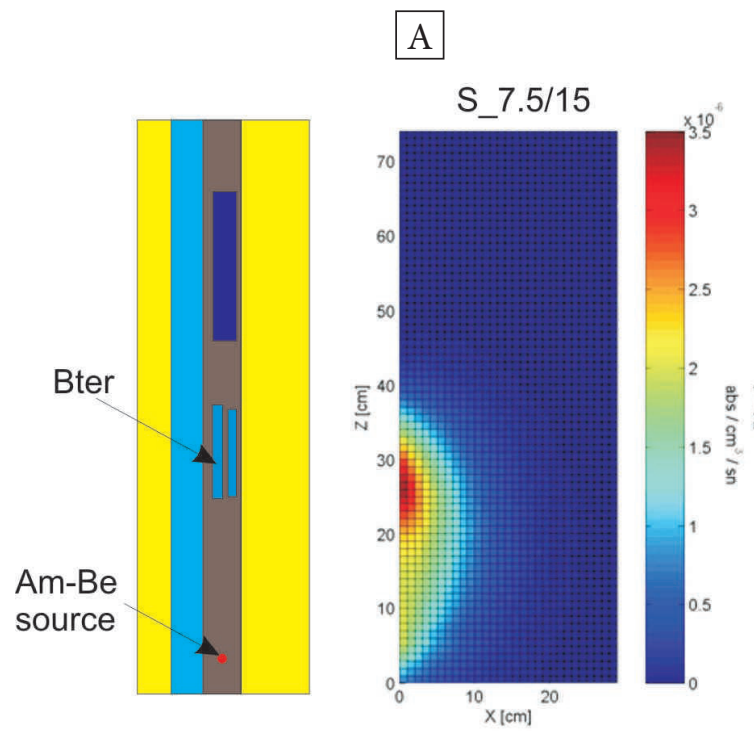

\section{B}

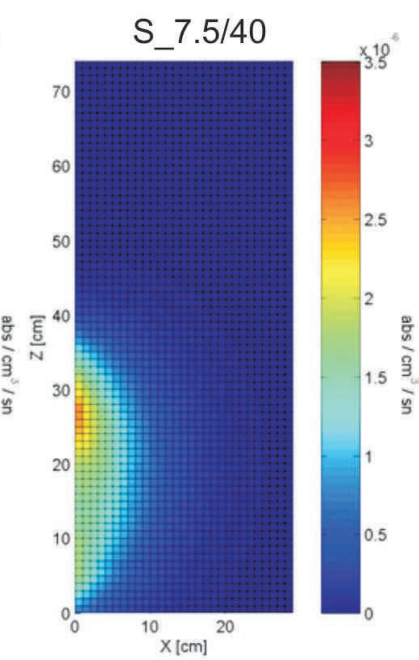

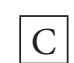

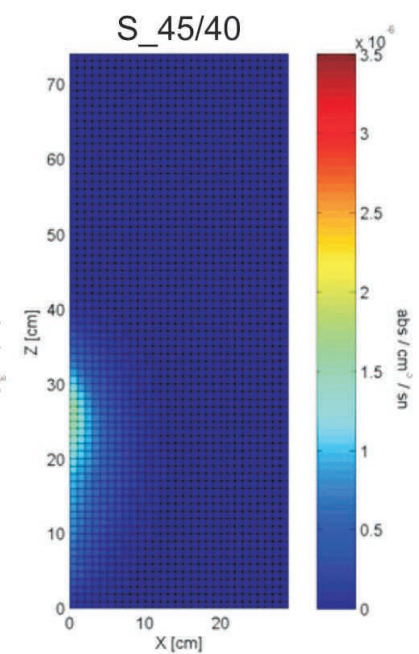

Fig. 10. Response of the "near" Bter detector in the Miocene models of different porosity $\varphi$ and $\Sigma_{\text {a rock: }}$ A) case $S_{-} 7.5 / 15$ : $\varphi=7.5 \%, \Sigma_{\text {a rock }}=15.54$ c.u.; B) case S_7.5/40: $\varphi=7.5 \%, \Sigma_{\text {a rock }}=38.67$ c.u.; C) case S_45/40: $\varphi=45 \%, \Sigma_{\text {a rock }}=32.01$ c.u.

The much more significant differences are shown when the porosity of the medium grows. This situation can be observed in Figure 10B and Figure 10C. The response of the Bter detector in the case S_45/40 drop to $50 \%$ in comparison to the case S_7.5/40 (Tab. 7, Fig. 9). For both cases (S_7.5/40 and S_45/40) the absorption cross sections do not differ too much. The main differences are in the neutron slow-down and diffusion length of neutrons. The porosity of Miocene S_7.5/40 is low, and in such a case is about $L_{\mathrm{s}}=15 \mathrm{~cm}$ and $L_{d}=7 \mathrm{~cm}$ (Czubek 1988). It means that the mean total distance of neutron travel is about $40 \mathrm{~cm}$. The porosity of Miocene S_45/40 is high ( $\varphi=45 \%)$, and the rock contains much more water (neutron moderator). Consequently $L_{s}$ is about $7 \mathrm{~cm}, L_{d}$ is about $3 \mathrm{~cm}$ and the mean total distance of neutron travel is about $20 \mathrm{~cm}$. 
The distance between the neutron source and the Bter detector is about $30 \mathrm{~cm}$. This explains the low probability of reaching the Bter detector by means of thermal neutrons. The example shows how important the optimization of the source-detector distance is.

The space distribution of epithermal neutrons which reach the detector Bepi (Fig. 11) is similar to the results for "near" thermal detector (Fig. 10). Only the number of counts is much higher in the detector Bepi than for the detector Bter what is obvious. In Figure 11 the space distribution of epithermal neutrons which reach the "near" detector Bepi is presented. The Miocene models: S_7.5/40 and S_45/40 differ in value of the absorption cross section only. The number of thermal neutrons reaching the detector does not depend on the thermal neutron absorption cross-section of medium. The number of counts in both cases is near the same (Tab. 7, Fig. 9). One can conclude that the maximum range, of the Bepi detector is also very similar in both cases.

Only significant differences are observed in the distribution of points from which the neutrons reach the detector. This effect may be due to the presence of a strong absorber in the model. A detailed analysis could be carried out on the basis of the distribution of neutron energy in the given space.

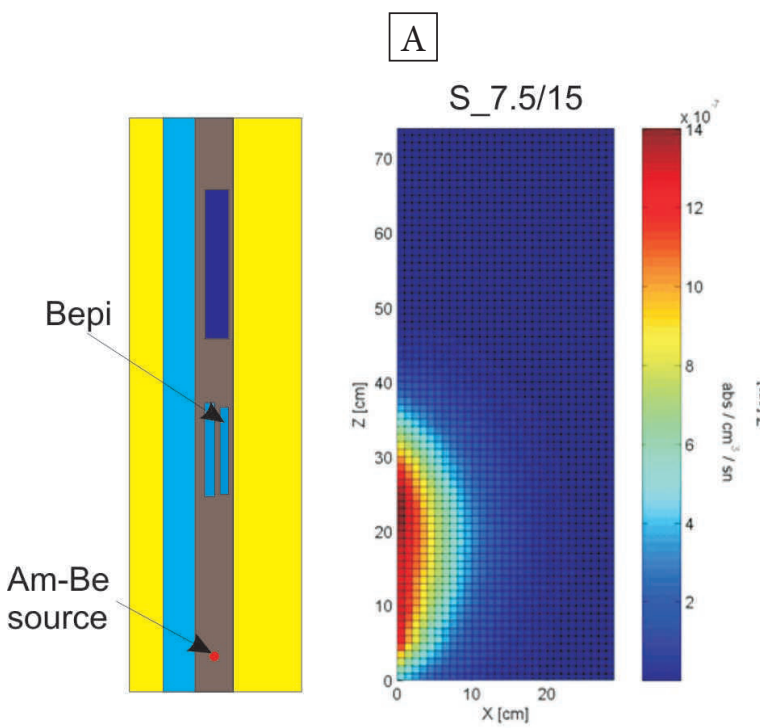

\section{B}

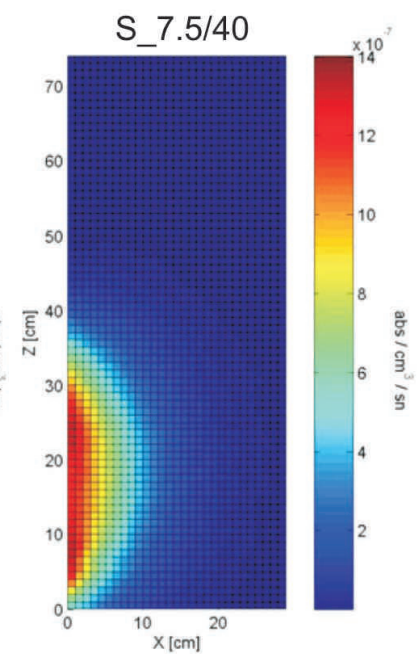

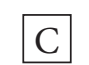

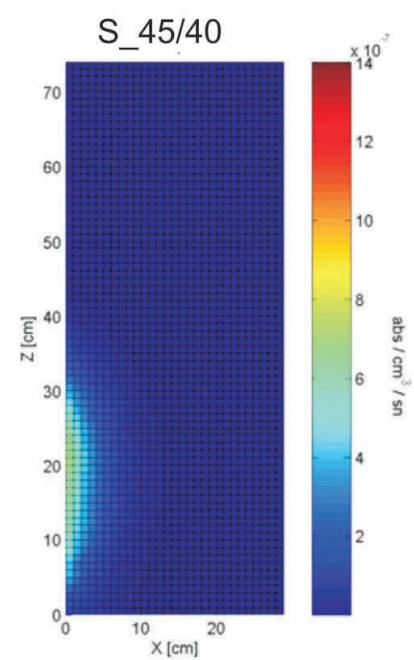

Fig. 11. Response of the "near" Bepi detector in the Miocene models of different porosity $\varphi$ and $\Sigma_{\text {a rock: }}$ A) case $S_{-} 7.5 / 15$ : $\varphi=7.5 \%, \Sigma_{\text {a rock }}=15.54$ c.u.; B) case S_7.5/40: $\varphi=7.5 \%, \Sigma_{\text {a rock }}=38.67$ c.u.; C) case S_45/40: $\varphi=45 \%, \Sigma_{\text {a rock }}=32.01$ c.u.

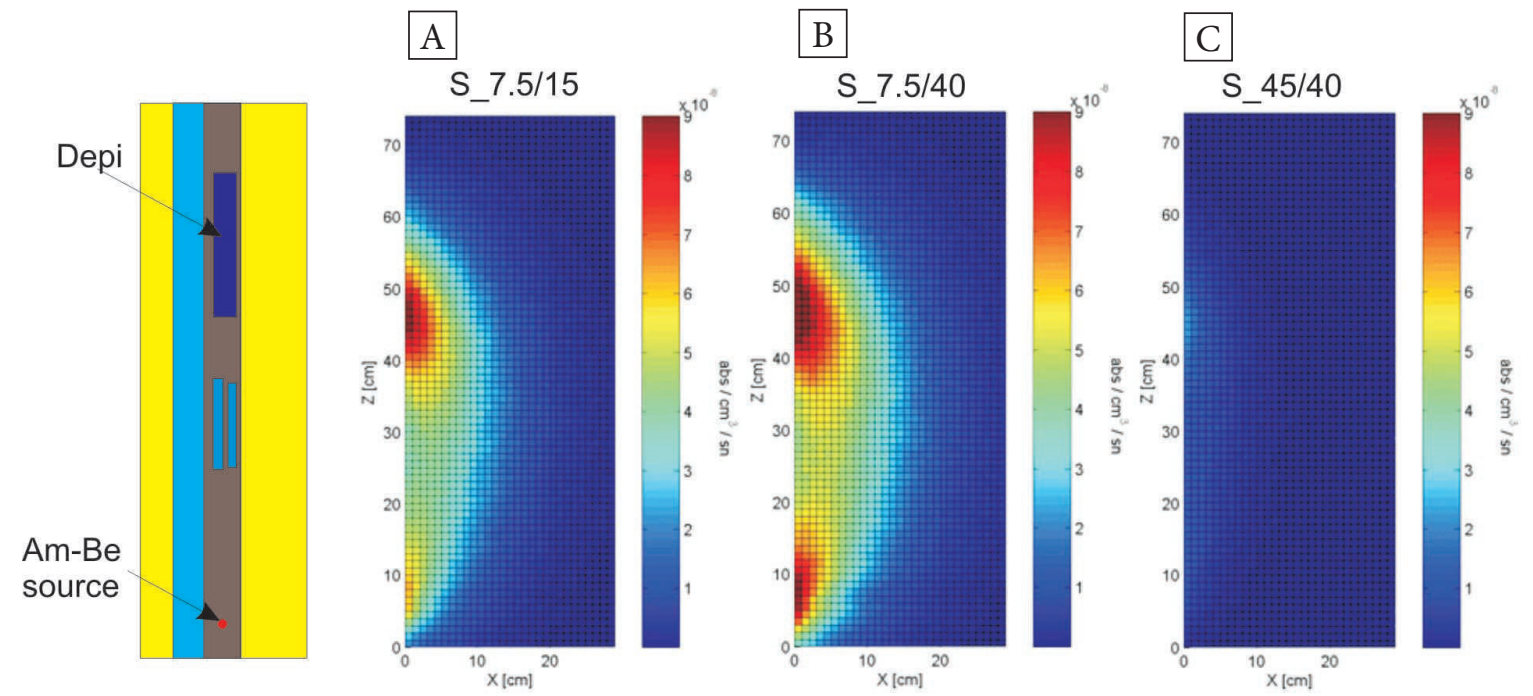

Fig. 12. Response of the "far" Depi detector in the Miocene models of different porosity $\varphi$ and $\left.\Sigma_{\text {a rock: }} A\right)$ case $S_{-} 7.5 / 15: \phi=7.5 \%$, $\Sigma_{\text {a rock }}=15.54$ c.u.; B) case S_7.5/40: $\phi=7.5 \%, \Sigma_{\text {a rock }}=38.67$ c.u.; C) case S_45/40: $\phi=45 \%, \Sigma_{\text {a rock }}=32.01$ c.u. 
These differences in the distribution shown for the cases: S_7.5/15 and S_7.5/40 in Figure 12 are of no importance when considering a homogeneous medium. The differences in the distribution of epithermal neutrons for Miocene S_45/40 with respect to the Miocene: S_7.5/15 and S_7.5/40 can be explained similarly as in the case of neutron distribution for the detector Bepi. A high porosity and water content means that the area of neutron migration is significantly decreased in relation to the medium with low water content.

\section{CONCLUSIONS}

The background possibilities of Monte Carlo modelling of the responses of the neutron well logging tool are presented in the paper. The NNTE borehole probe was chosen to introduce the computational capabilities of the MCNP program. Numerical modelling using the MCNP code allows us to track the history of neutron exiting from the source until its absorption in the medium. This process can be completed in complex 3D geometry containing a probe, borehole and surrounding medium. The neutron can, both when leaving the source and where it reaches the detector, can react not only with the borehole fluid and geological layer but also with the mechanical elements of the probe. In that reason the whole mechanical construction of the tool has to be numerically modelled in detail. The MCNP code gives us such an opportunity, so the actual physical experiment can be faithfully reproduced numerically.

The benchmark calculations presented in the paper confirmed the very good reproducibility of the real measurements taken by the NNTE probe. The simulations confirmed the need to know in detail the elemental composition of the entire modeled system. In order to have fully reliable simulation results for thermal neutron, the presence of strong neutron absorbers $(\mathrm{B}, \mathrm{Cl}$, rare earths) must be known to an accuracy of a few $\mathrm{ppm}$. For the purposes of an interpretation of the NNTE readings an issue of the strong thermal neutron absorbents comes down to knowledge of the absorption cross section $\Sigma_{a}$. For a given bulk material $\Sigma_{a}$ can be calculated from its elemental composition. It seems to be easy to implement, but note that it requires the determination of small impurities B, C, rare earths at a level of few ppm which can significantly increase the cost of analysis. $\Sigma_{a}$ can be measured experimentally on samples or, in the case of NNTE probe may also be determined on the basis of an adequate analysis of the detector responses. The MCNP simulations can be used for a detailed analysis of the measurements carried on the NNTE probe in a wide range of $\Sigma_{a}$ variation.

The paper also presents the possibility to visualize the spatial distribution of neutrons using a Monte Carlo simulation of a neutron migration in bulk media. Our aim was to show the graphical representation of which areas and in what amounts neutrons reach each of the detector probes. The examples show which areas (points) considered the center reach the neutron detector and the extent of penetration of the neutron probe is estimated. It is known that many of the neutrons produced by a neutron source are absorbed in the media and only a part of them reached and is detected in the volume of detector.

This method of presentation may be useful for the visualization of the spatial and energy distributions of neutrons for more complex systems, e.g. for thin-layer formations, for systems with an invaded zone near a borehole, etc.

The following examples of distributions are one of many possible implementations of the visual field of a neutron originating from the target neutron source. There is the possibility to create maps of neutron energy distribution, and in the event of pulsed neutron sources, e.g. of time distributions of neutrons.

We have shown that the MCNP software enables a wide range of applications of computer simulations to nuclear well logging methods. The program can be used not only to simulate neutron-transport in a given bulk media with a complex geometry, but also to simulate the gamma rays and other charged particles - electrons, protons, alpha particles, etc. It extends the possibilities of using this software to simulate different variants of the probes with gamma sources.

The authors would like to express their gratitude to Msc. Barbara Gaban'ska, for her help and valuable comments during the work. 


\section{REFERENCES}

Cywicka-Jakiel T., Woźnicka U. \& Zorski T., 2006. Wpływ zweryfikowanego składu pierwiastkowego wzorców skalnych i nowej biblioteki danych neutronowych na funkcję odpowiedzi spektrometrycznej sondy neutron-gamma. Prace Instytutu Nafty i Gazu, 137, 523-529.

Czubek J.A., 1988. Półempiryczna kalibracja sond neutronowych (Przybliżenie jedno- $i d w u$-grupowe). Raport IFJ No. 1413/AP, Kraków.

Drabina A., Zorski T. \& Woźnicka U., 2003. Correlation between Measurements and Monte-Carlo Calculations for the NNTE Logging-Tool. INP Report No 1926/AP, [on-line:] www.ifj.edu.pl/reports/2003.html [access: 28.04.2016].

Drabina A. \& Zorski Z., 2005. Geophysical Neutron Logging Tool NNTE for Measurements of Porosity and Rock Matrix Sigma_a - Numerical Simulations. [in:] Proceedings of the enlargement workshop on Neutron Measurements, Evaluations and Applications-2, NEMEA-2, 20-23 October 2004, Bucharest, Romania, European Communities, 81-84.

Herman M. \& Trkov A., 2009. ENDF-6 Formats Manual Data Formats and Procedures for the Evaluated Nuclear Data Files ENDF/B-VI and ENDF/B-VII. Brookhaven National Laboratory Report BNL-90365-2009 Rev. 1.

Klüge H., 1998. Irradiation Facility with Radioactive Reference Neutron Sources: Basic Principles. PTB-Bericht N-34, Braunschweig.
The MathWorks Inc., 2012. MATLAB_ The Language of Technical Computing User's Manual. [on-line:] http:// www.mathworks.com/help/matlab/index.html [access: 28.04.2016]

MCNP team, 2008. X-5 Monte Carlo Team, MCNP - A General Monte Carlo N-Particle Transport Code Version 5. Los Alamos National Laboratory, LA-UR-03-1987.

Mughabghab S.F., 1984. Neutron Cross Sections: Neutron resonance parameters and thermal cross sections Part $A$ and Part B. Academic Press, Orlando.

Woźnicka U., Dworak D., Wiącek U. \& Zorski T., 2012. Geofizyczne profilowania neutronowe $w$ asymetrycznych formacjach cienkowarstwowych. Symulacje komputerowe na przykładzie sondy NNTE. IFJ PAN, Kraków.

Zorski T., Massalski T., Drabina A. \& Stadtmüller M., 1996. Metodyka pomiarów kalibracyjnych i standaryzacyjnych dla neutronowych sond geofizyki wiertniczej w Zakładzie Geofizyka - Kraków. Nafta-Gaz, 11, 493-505.

Zorski T. \& Stadtmüller M., 2001. Opracowanie alternatywnej w stosunku do profilowania gamma (PG) metody wyznaczania zasilenia $w$ cienkowarstwowych formacjach miocenu przedgórza Karpat na podstawie pomiarów sonda neutronowa mierzaca jednocześnie neutrony termiczne i nadtermiczne [internal report of research project No. 5.5.140.771, sponsored by the Polish Oil and Gas Company]. Akademia Górniczo-Hutnicza, Wydział Geologii, Geofizyki i Ochrony Środowiska, Kraków. 